\title{
Tidal effects for spinning particles
}

\author{
Rafael Aoude, ${ }^{a}$ Kays Haddad $^{b}$ and Andreas Helset ${ }^{c}$ \\ ${ }^{a}$ Centre for Cosmology, Particle Physics and Phenomenology (CP3), \\ Université catholique de Louvain, \\ 1348 Louvain-la-Neuve, Belgium \\ ${ }^{b}$ Niels Bohr International Academy and Discovery Center, Niels Bohr Institute, \\ University of Copenhagen, \\ Blegdamsvej 17, DK-2100 Copenhagen, Denmark \\ ${ }^{c}$ Walter Burke Institute for Theoretical Physics, California Institute of Technology, \\ Pasadena, CA 91125, U.S.A. \\ E-mail: rafael.aoude@uclouvain.be, kays.haddad@nbi.ku.dk, \\ andreas.helset@caltech.edu
}

AbStRACT: Expanding on the recent derivation of tidal actions for scalar particles, we present here the action for a tidally deformed spin-1/2 particle. Focusing on operators containing two powers of the Weyl tensor, we combine the Hilbert series with an on-shell amplitude basis to construct the tidal action. With the tidal action in hand, we compute the leading-post-Minkowskian tidal contributions to the spin-1/2-spin-1/2 amplitude, arising at $\mathcal{O}\left(G^{2}\right)$. Our amplitudes provide evidence that the observed long range spin-universality for the scattering of two point particles extends to the scattering of tidally deformed objects. From the scattering amplitude we find the conservative two-body Hamiltonian, linear and angular impulses, eikonal phase, spin kick, and aligned-spin scattering angle. We present analogous results in the electromagnetic case along the way.

KEYwords: Effective Field Theories, Scattering Amplitudes, Black Holes

ArXiv EPrint: 2012.05256 


\section{Contents}

1 Introduction 1

2 Tidal actions 3

2.1 Hilbert series 3

2.2 Amplitude basis for QED 4

2.3 Amplitude basis for gravity 6

2.4 Operator basis for QED 6

$\begin{array}{ll}2.5 & \text { Operator basis for gravity }\end{array}$

3 Leading-PM tidal effects $\quad 9$

$\begin{array}{lll}3.1 & \text { QED } & 10\end{array}$

3.2 Gravity 12

4 Conservative two-body Hamiltonian $\quad 15$

5 Classical observables $\quad 18$

$\begin{array}{lll}5.1 & \text { Linear impulse } & 18\end{array}$

$\begin{array}{ll}5.2 & \text { Angular impulse } \\ \end{array}$

6 Eikonal phase $\quad 21$

$\begin{array}{lll}7 & \text { Conclusion } & 24\end{array}$

$\begin{array}{ll}\text { A Loop integrals } & 25\end{array}$

B Integrals for classical impulses and the potential 29

$\begin{array}{lll}\text { B.1 Fourier transforms } & 29\end{array}$

B.2 Linear and angular impulse 30

B.3 Eikonal operator 32

\section{Introduction}

The surge of attention payed to the binary inspiral problem in general relativity (GR) by the scattering amplitudes community - caused by the observation of gravitational waves by the LIGO/Virgo collaborations [1] — has produced a greater understanding of the description of classical properties of binary systems using quantum-field-theoretic and amplitudes techniques. To date, most of the work has focused on point particles. Some notable results in this direction include elucidating methods for converting scattering amplitudes for relativistic point particles to classical observables [2-11], the description of 
classical angular momentum from quantum mechanical spin [2, 6, 8, 12-19], and the stateof-the-art computation of the third post-Minkowskian (3PM) dynamics of a spinless binary system [20-23]. Despite tremendous progress, there remains much to be understood about the connection between scattering amplitudes and classical binary systems.

The quantum description of tidal effects is a topic of particular interest of late. While Schwarzschild black holes do not tidally deform in four spacetime dimensions [24-27], there is still debate whether the same is true for Kerr black holes in a general gravitational environment [28-31]. Nevertheless, such effects impact the gravitational wave signal of a neutron star merger $[32,33]$, so understanding them is necessary for the full description of these systems. The study of such effects from a scattering amplitudes perspective was only recently initiated in ref. [34]. There, the authors included higher-dimensional operators quadratic in the Weyl tensor in the action of a gravitating scalar particle, and computed the corrections to the Hamiltonian and scattering angle up to next-to-leading post-Minkowskian (PM) order $\left(\mathcal{O}\left(G^{3}\right)\right.$ for tidal effects). Not long after, two of the present authors applied the Hilbert series to extend the action of ref. [34] to include a complete, non-redundant set of operators quadratic in the Weyl tensor [35]. They subsequently computed all $\mathcal{O}\left(G^{2}\right)$ finite-size ${ }^{1}$ contributions to scalar-scalar scattering, including all contributions from higher-derivative operators. Since then, refs. [36, 37] extended the study of finite-size effects by calculating the leading-PM contributions from an infinite set of operators with higher powers of the Weyl tensor, using the geodesic equation and unitarity cuts, respectively.

There have also been efforts to derive fully relativistic information about tidally deformed systems from purely classical frameworks. Refs. [38, 39] incorporated tidal effects into PM worldline actions, and subsequently derived the leading-PM contributions to observables from a subset of tidal effects arising from couplings quadratic in the Weyl tensor. Ref. [40] extended these results to a larger subset of these tidal operators, as well as to the next-to-leading PM order. Incorporating spin, refs. [41, 42] extended the operator basis of ref. [43] to include operators quadratic in the curvature containing up to four powers of the spin vector. Most recently, ref. [44] presented a relativistic action describing tidally deformed bodies up to linear order in spin.

To date, there has been no amplitudes approach adding spin to the tidally deformed object. In this paper we fill this gap by expanding on the work of ref. [35] to include spin effects. Combining the Hilbert series with on-shell methods, we construct the full action for spinors and two powers of the Weyl tensor. This allows us to compute all classical tidal effects at $\mathcal{O}\left(G^{2}\right)$ for spinor-spinor scattering. Adapting the spinning effective field theory matching of ref. [8], we present the interaction Hamiltonian including spin and tidal effects at $\mathcal{O}\left(G^{2}\right)$ and to linear order in the angular momentum of each body. Then, applying the methods of refs. $[4,6]$, we use the amplitudes to find the linear and angular impulses. We present the analogous results for quantum electrodynamics (QED). We then compute the eikonal phase to extract additional observables in the gravitational case. First, the

\footnotetext{
${ }^{1}$ We will use the terms "tidal" and "finite-size" interchangably for both gravity and electromagnetism, even though "electric/magnetic susceptibility" is normally used in the electromagnetic context.
} 
eikonal phase allows us to verify the linear impulse through a separate method. Then, it provides a means for computing the spin kick and the tidal corrections to the aligned-spin scattering angle.

This paper is organized as follows. We start by finding the tidal actions in both the electromagnetic and gravitational cases for a massive spin- $1 / 2$ particle in section 2 . This is accomplished by first using the Hilbert series in section 2.1, which provides a guide for finding the amplitude bases in sections 2.2 and 2.3 and the operator bases in sections 2.4 and 2.5. We then calculate the leading-PM scattering amplitudes in section 3. The scattering amplitudes are then used to calculate various classical quantities: the conservative Hamiltonian in section 4, linear and angular impulses in section 5, and the eikonal phase, spin kick, and aligned-spin scattering angle in section 6. We conclude in section 7. Appendix A contains a discussion on the relevant loop integrals, while in appendix B we show details for the calculation of classical observables.

\section{Tidal actions}

Combining the Hilbert series with on-shell amplitudes methods, we construct in this section the full action coupling two photon field strengths or two Weyl tensors to spinor fields.

\subsection{Hilbert series}

We begin with the Hilbert series. The Hilbert series produces the number of group invariants for a given field content, and it is useful when constructing an operator basis in an effective field theory. Notable achievements are the applications of the Hilbert series to the Standard Model effective field theory [45-49], and the extension to include gravity [50]. Non-relativistic effective field theories $[51,52]$ and effective field theories with non-linearly realized symmetries [53] can also be constructed using Hilbert series techniques.

The Hilbert series was applied to characterize tidal effects for post-Minkowskian scattering in ref. [35]. In addition to the structures described in appendix A of ref. [35], we need the group characters for left- and right-handed Weyl spinors (respectively $\psi$ and $\psi^{\dagger}$ ) [50],

$$
\begin{aligned}
& \chi_{[3 / 2,(1 / 2,0)]}(\mathcal{D} ; x, y)=\mathcal{D}^{3 / 2} P(\mathcal{D} ; x, y)\left[\chi_{(1 / 2,0)}(x, y)-\mathcal{D} \chi_{(0,1 / 2)}(x, y)\right], \\
& \chi_{[3 / 2,(0,1 / 2)]}(\mathcal{D} ; x, y)=\mathcal{D}^{3 / 2} P(\mathcal{D} ; x, y)\left[\chi_{(0,1 / 2)}(x, y)-\mathcal{D} \chi_{(1 / 2,0)}(x, y)\right] .
\end{aligned}
$$

Moreover, in both the electromagnetic and gravitational cases, we assume the spinor fields are charged under a $\mathrm{U}(1)$ gauge group. Thus we also need the gauge group characters $\chi_{\mathrm{U}(1)}(\alpha)=\alpha^{Q}$ for a particle with charge $Q$ and the corresponding Haar measure:

$$
\int d \mu_{\mathrm{U}(1)}=\frac{1}{2 \pi i} \oint_{|\alpha|=1} \frac{d \alpha}{\alpha}
$$

All other relevant information is given in ref. [35]. 
We can now compute the Hilbert series in which we are interested. The Hilbert series for two field strengths coupled to spinors for mass dimension $d, \mathcal{H}_{d}^{F^{2}}$, is

$$
\begin{aligned}
\mathcal{H}_{7+2 n}^{F^{2}}= & \lfloor n / 2+1\rfloor\left(F_{L}^{2}+F_{R}^{2}\right)\left(\psi \psi^{c}+\psi^{c \dagger} \psi^{\dagger}\right) D^{2 n}+n F_{L} F_{R}\left(\psi \psi^{c}+\psi^{c \dagger} \psi^{\dagger}\right) D^{2 n} \\
& +\frac{1}{2}\left(1-(-1)^{n}\right)\left(F_{L}^{2} \psi \psi^{c}+F_{R}^{2} \psi^{c \dagger} \psi^{\dagger}\right) D^{2 n}, \\
\mathcal{H}_{6+2 n}^{F^{2}}= & \lfloor n / 2\rfloor\left(F_{L}^{2}+F_{R}^{2}\right)\left(\psi \psi^{\dagger}+\psi^{c} \psi^{c \dagger}\right) D^{2 n-1}+n F_{L} F_{R}\left(\psi \psi^{\dagger}+\psi^{c} \psi^{c \dagger}\right) D^{2 n-1} .
\end{aligned}
$$

In eq. (2.4) we have $n \geq 0$, whereas $n \geq 1$ in eq. (2.5). Coupling two Weyl tensors to spinors, the Hilbert series for mass dimension $d, \mathcal{H}_{d}^{C^{2}}$, is

$$
\begin{aligned}
\mathcal{H}_{7+2 n}^{C^{2}}= & \lfloor n / 2+1\rfloor\left(C_{L}^{2}+C_{R}^{2}\right)\left(\psi \psi^{c}+\psi^{c \dagger} \psi^{\dagger}\right) D^{2 n}+(n-1) C_{L} C_{R}\left(\psi \psi^{c}+\psi^{c \dagger} \psi^{\dagger}\right) D^{2 n} \\
& +\frac{1}{2}\left(1-(-1)^{n}\right)\left(C_{L}^{2} \psi \psi^{c}+C_{R}^{2} \psi^{c \dagger} \psi^{\dagger}\right) D^{2 n}, \\
\mathcal{H}_{6+2 n}^{C^{2}}= & \lfloor n / 2\rfloor\left(C_{L}^{2}+C_{R}^{2}\right)\left(\psi \psi^{\dagger}+\psi^{c} \psi^{c \dagger}\right) D^{2 n-1}+(n-1) C_{L} C_{R}\left(\psi \psi^{\dagger}+\psi^{c} \psi^{c \dagger}\right) D^{2 n-1} .
\end{aligned}
$$

Once again, $n \geq 0$ in eq. (2.6) and $n \geq 1$ in eq. (2.7).

Eqs. (2.5) and (2.7) are the Hilbert series for even mass dimensions. These operators do not have any analogs in the complex scalar case (which is a slight generalization of the real scalar case discussed in ref. [35]), and we will see that they all contribute spin effects in the PM amplitudes.

The Hilbert series for odd mass dimensions in eqs. (2.4) and (2.6) are very similar to the corresponding Hilbert series for complex scalars coupled to photons or gravitons, respectively (up to a doubling of the number of terms coming from chiral fermions). The main difference is the appearance of the additional term

$$
\frac{1}{2}\left(1-(-1)^{n}\right)\left(F_{L}^{2} \psi \psi^{c}+F_{R}^{2} \psi^{c \dagger} \psi^{\dagger}\right) D^{2 n},
$$

or its analog for gravitons. These terms are present for $d=9,13,17, \ldots$ - where they contribute spin effects - but are absent for $d=11,15,19, \ldots$ In the next section, we will see that this curious behavior can be understood using on-shell spinor-helicity variables.

\subsection{Amplitude basis for QED}

A complementary approach to the characterization of tidal effects is the construction of on-shell amplitudes. It will be useful to us as it will elucidate relations among operators that are not obvious in an off-shell language. The massive spinor-helicity formalism of ref. [54] is ideal for our purposes, and we will make use of it to construct the on-shell amplitude basis. The massive spinors are indicated by a bolding of the momentum labels, which also represents a symmetrization over the massive particle's little group indices. See refs. [55-58] for recent work constructing on-shell amplitudes.

Our approach makes use of the spinor structures presented in ref. [55]. To extend these results to higher mass dimensions, the various spinor structures are multiplied by combinations of Mandelstam variables, $s_{i j} \equiv\left(p_{i}+p_{j}\right)^{2}$, in a way that respects the Bose/Fermi statistics of the system. At four-points there are two independent Mandelstam variables. 


\begin{tabular}{|c|c|}
\hline Helicity & Amplitude \\
\hline$(++++)$ & {$[12]([14][23]+[13][24]) y^{2 b+1}$} \\
\hline$(++--)$ & {$[12]^{2}\langle\mathbf{3 4}\rangle x^{a} y^{2 b}$} \\
\hline$(+-++)$ & {$[1|(\mathbf{3}-\mathbf{4})| 2\rangle^{2}[\mathbf{3 4}] x^{a} y^{b}$} \\
\hline$(+++-)$ & {$[12]^{2}[\mathbf{3}|(1-2)| \boldsymbol{4}\rangle x^{a} y^{2 b+1}$} \\
\hline$(++-+)$ & {$[12]^{2}\langle\mathbf{3}|(1-2)| \mathbf{4}] x^{a} y^{2 b+1}$} \\
\hline$(+-+-)$ & {$[1 \mathbf{3}]\langle 2 \mathbf{4}\rangle[1|(\mathbf{3}-\mathbf{4})| 2\rangle x^{a} y^{b}$} \\
\hline
\end{tabular}

Table 1. The amplitude basis for electromagnetic finite-size effects. The helicity labels are ordered as $\left(\gamma_{1} \gamma_{2} \psi_{3} \psi_{4}\right)$. The three first rows are the amplitude basis for odd mass dimensions, while the three last rows are for even mass dimensions. Here $a$ and $b$ take integer values from 0 to $\infty$. The amplitudes for opposite helicities can be obtained by exchanging angle and square brackets.

Labeling the two bosons as 1 and 2 and the two fermions as 3 and 4, we work with the two combinations of Mandelstam variables $x=s_{12}$ and $y=s_{13}-s_{23}+s_{24}-s_{14} .^{2}$ These combinations manifest symmetry/antisymmetry under the separate exchanges $1 \leftrightarrow 2$ and $3 \leftrightarrow 4$.

We generate all higher-dimensional helicity amplitudes by multiplying the various spinor structures by products of Mandelstam variables, e.g. $x^{a} y^{b}$ for $a, b$ non-negative integers. As we are interested in amplitudes for two bosons and two spinors, all amplitude structures must be symmetric (antisymmetric) under the exchange $1 \leftrightarrow 2(3 \leftrightarrow 4)$ for amplitudes with indistinguishable particles. While Bose symmetry allows the power of $x$ in a helicity amplitude to be arbitrary, it restricts the power of $y$ in certain amplitudes to be either even or odd, i.e. some spinor structures will be multiplied by $x^{a} y^{2 b(+1)}$. Finally, ref. [56] argued that spinor structures for massless particles can be generalized to the massive case by simply bolding the momentum labels of the massive spin- $1 / 2$ particles. All things considered, the amplitude basis for two massive spinors coupled to two photons is given in table 1.

We are now in a position to discuss the curious operators counted in eq. (2.8). They correspond to the second helicity amplitude in the first row of table 1 . The reason why they are only present for $d=9,13,17, \ldots$ is a special relation between the helicity amplitudes. The helicity amplitude structures (considering massless fermions for simplicity)

$$
[12]^{2}[34] x^{a} y^{2 b} \quad \text { and } \quad[12]([14][23]+[13][24]) y^{2 c+1}
$$

are independent for any $a, b, c$. However, if we multiply the second helicity amplitude in eq. (2.9) by $x$, then we obtain the relation

$$
2[12]([14][23]+[13][24]) x y^{2 c+1}=-[12]^{2}[34] y^{2 c+2} .
$$

\footnotetext{
${ }^{2}$ In massive four-point amplitudes, we must include the mass of the fermions as a further independent structure. However, the mass can always be absorbed into a Wilson coefficient, changing the dimensionality of the amplitude under consideration. Therefore, at a fixed mass dimension, it is sufficient to construct the helicity amplitudes using only $x$ and $y$.
} 


\begin{tabular}{|c|c|}
\hline Helicity & Amplitude \\
\hline$(++++)$ & {$[12]^{3}([1 \mathbf{4}][2 \mathbf{3}]+[1 \mathbf{3}][24]) y^{2 b+1}$} \\
\hline$(++--)$ & {$[12]^{4}\langle\mathbf{3 4}\rangle x^{a} y^{2 b}$} \\
\hline$(+-++)$ & {$[1|(\mathbf{3}-\mathbf{4})| 2\rangle^{4}[\mathbf{3 4}] x^{a} y^{b}$} \\
\hline$(+++-)$ & {$[12]^{4}[\mathbf{3}|(1-2)| \mathbf{4}\rangle x^{a} y^{2 b+1}$} \\
\hline$(++-+)$ & {$[12]^{4}\langle\mathbf{3}|(1-2)| \mathbf{4}] x^{a} y^{2 b+1}$} \\
\hline$(+-+-)$ & {$[13]\langle 24\rangle[1|(\mathbf{3}-\mathbf{4})| 2\rangle^{3} x^{a} y^{b}$} \\
\hline
\end{tabular}

Table 2. The amplitude basis for gravitational tidal effects. The helicity labels are ordered as $\left(g_{1} g_{2} \psi_{3} \psi_{4}\right)$. The three first rows are the amplitude basis for odd mass dimensions, while the three last rows are for even mass dimensions. Here $a$ and $b$ take integer values from 0 to $\infty$. The amplitudes for opposite helicities can be obtained by exchanging angle and square brackets.

In the massive case this equivalence is modified by a lower-dimensional spinor structure. Thus, we can choose an amplitude basis where the second term in eq. (2.9) is never multiplied by $x$. Note that the analogous relation holds for gravitons, where each helicity amplitude is multiplied by $[12]^{2}$. The relation remains true when exchanging the square brackets for angle brackets.

\subsection{Amplitude basis for gravity}

The amplitude basis for gravity is almost identical to the photon case, with some additional powers of [12], $\langle 1|(\mathbf{3}-\mathbf{4})| 2]$, or their conjugates, accounting for the additional little group weights of gravitons relative to photons. The full amplitude basis for gravity is listed in table 2 .

\subsection{Operator basis for QED}

With the explicit amplitude basis at hand, we can turn to finding the corresponding operator basis. It can be beneficial to have both an amplitude and an operator basis, since then both on-shell and off-shell calculations can be performed directly starting from the appropriate basis. Either approach can be used to calculate leading or subleading PM amplitudes.

In our case, the amplitude basis serves as a guide and as a cross-check. The products of Mandelstam variables correspond to the distribution of covariant derivatives in the operators, and the spinor structure can be simply found by putting various operators onshell. Moreover, the relation in eq. (2.10) indicates a relation between off-shell operators that we must take into account. As a cross-check, we have verified that the operator basis below matches the amplitude basis in table 1 when put on-shell.

The full Lagrangian for fermions coupled to two field strengths is

$$
\mathcal{L}_{\mathrm{QED}}=\bar{\psi}(i \not D-m) \psi+\Delta \mathcal{L}_{\mathrm{QED}}^{\mathrm{odd}}+\Delta \mathcal{L}_{\mathrm{QED}}^{\mathrm{even}}
$$

where $\Delta \mathcal{L}_{\mathrm{QED}}^{\text {odd/even }}$ are the contributions from higher-dimensional operators at odd or even mass dimensions, respectively. Throughout this paper, we will use the prefix $\Delta$ to denote 
tidal contributions, unless otherwise stated. The contribution to the Lagrangian at odd mass dimensions is

$$
\begin{aligned}
& \Delta \mathcal{L}_{\mathrm{QED}}^{\text {odd }}=\sum_{n=0}^{\infty} \sum_{k=0}^{\lfloor n / 2\rfloor} a_{1}^{(n, k)}\left(\bar{\psi} \stackrel{\leftrightarrow}{D^{\alpha_{1} \ldots \alpha_{2 k}} \psi}\right)\left(D_{\beta_{1} \ldots \beta_{n-2 k}} F^{\mu \nu} \stackrel{\leftrightarrow}{D}_{\alpha_{1} \ldots \alpha_{2 k}} D^{\beta_{1} \ldots \beta_{n-2 k}} F_{\mu \nu}\right) \\
& +\sum_{n=0}^{\infty} \sum_{k=0}^{\lfloor n / 2\rfloor} a_{2}^{(n, k)}\left(\bar{\psi} \stackrel{\leftrightarrow}{D}{ }^{\mu \nu \alpha_{1} \ldots \alpha_{2 k}} \psi\right)\left(D_{\beta_{1} \ldots \beta_{n-2 k}} F_{\mu} \stackrel{\leftrightarrow}{D}_{\alpha_{1} \ldots \alpha_{2 k}} D^{\beta_{1} \ldots \beta_{n-2 k}} F_{\nu \rho}\right) \\
& +\sum_{n=0}^{\infty} \sum_{k=0}^{\lfloor n / 2\rfloor} i a_{3}^{(n, k)}\left(\bar{\psi} \gamma_{5} \stackrel{\leftrightarrow}{D} \mu \nu \alpha_{1} \ldots \alpha_{2 k+1} \psi\right)\left(D_{\beta_{1} \ldots \beta_{n-2 k}} F_{\mu} \stackrel{\leftrightarrow}{D}_{\alpha_{1} \ldots \alpha_{2 k+1}} D^{\beta_{1} \ldots \beta_{n-2 k}} \tilde{F}_{\nu \rho}\right) \\
& +\sum_{n=0}^{\infty} \sum_{k=0}^{\lfloor n / 2\rfloor} i a_{4}^{(n, k)}\left(\bar{\psi} \gamma_{5} \stackrel{\leftrightarrow}{D^{\alpha} \ldots \alpha_{2 k}} \psi\right)\left(D_{\beta_{1} \ldots \beta_{n-2 k}} F^{\mu \nu} \stackrel{\leftrightarrow}{D}_{\alpha_{1} \ldots \alpha_{2 k}} D^{\beta_{1} \ldots \beta_{n-2 k}} \tilde{F}_{\mu \nu}\right) \\
& +\sum_{n=0}^{\infty} i b^{(n)}\left(\bar{\psi} \sigma^{\mu \nu} \stackrel{\leftrightarrow}{D}^{\rho \alpha_{1} \ldots \alpha_{2 n}} \psi\right)\left(F_{\mu \rho} \stackrel{\leftrightarrow}{D}_{\sigma \alpha_{1} \ldots \alpha_{2 n}} F_{\nu}{ }^{\sigma}\right)
\end{aligned}
$$

We have only included parity-even operators. We have used the short-hand notation $D^{\mu_{1} \ldots \mu_{k}}=D^{\mu_{1}} \ldots D^{\mu_{k}}$ and $A \stackrel{\leftrightarrow}{D^{\mu}} B=A\left(D^{\mu} B\right)-\left(D^{\mu} A\right) B$. In particular, $A \stackrel{\leftrightarrow}{D}{ }^{\mu_{1} \ldots \mu_{k}} B=$ $A \stackrel{\leftrightarrow}{D}^{\mu_{1} \ldots \mu_{k-1}}\left(D^{\mu_{k}} B\right)-\left(D^{\mu_{k}} A\right) \stackrel{\leftrightarrow}{D} \mu_{1} \ldots \mu_{k-1} B+\mathcal{O}\left(F^{3}\right)$. For our purposes, we only need the part quadratic in the field strengths or Weyl tensors.

The operators labelled by the Wilson coefficient $b^{(n)}$ produce the on-shell structure on the right of eq. (2.9). As a consequence of eq. (2.10), these operators only arise at every second odd mass dimension.

The contribution at even mass dimensions is

$$
\begin{aligned}
\Delta \mathcal{L}_{\mathrm{QED}}^{\text {even }}= & \sum_{n=0}^{\infty} \sum_{k=0}^{\lfloor n / 2\rfloor} i c_{1}^{(n, k)}\left(\bar{\psi} \gamma_{\mu} \stackrel{\leftrightarrow}{D}{ }^{\nu \alpha_{1} \ldots \alpha_{2 k}} \psi\right)\left(D_{\beta_{1} \ldots \beta_{n-2 k}} F^{\mu \rho} \stackrel{\leftrightarrow}{D}_{\alpha_{1} \ldots \alpha_{2 k}} D^{\beta_{1} \ldots \beta_{n-2 k}} F_{\nu \rho}\right) \\
& +\sum_{n=0}^{\infty} \sum_{k=0}^{\lfloor n / 2\rfloor} i c_{2}^{(n, k)}\left(\bar{\psi} \gamma_{\mu} \stackrel{\leftrightarrow}{D} \nu \lambda \alpha_{1} \ldots \alpha_{2 k} \psi\right)\left(D_{\beta_{1} \ldots \beta_{n-2 k}} F^{\mu \rho} \stackrel{\leftrightarrow}{D}_{\lambda \alpha_{1} \ldots \alpha_{2 k}} D^{\beta_{1} \ldots \beta_{n-2 k}} F_{\nu \rho}\right) \\
& +\sum_{n=0}^{\infty} \sum_{k=0}^{\lfloor n / 2\rfloor} c_{3}^{(n, k)}\left(\bar{\psi} \gamma_{5} \gamma_{\mu} \stackrel{\leftrightarrow}{D}{ }^{\nu \lambda \alpha_{1} \ldots \alpha_{2 k}} \psi\right)\left(D_{\beta_{1} \ldots \beta_{n-2 k}} F^{\mu \rho} \stackrel{\leftrightarrow}{D}_{\lambda \alpha_{1} \ldots \alpha_{2 k}} D^{\beta_{1} \ldots \beta_{n-2 k}} \tilde{F}_{\nu \rho}\right. \\
& \left.-D_{\beta_{1} \ldots \beta_{n-2 k}} \tilde{F}^{\mu \rho} \stackrel{\leftrightarrow}{D}_{\lambda \alpha_{1} \ldots \alpha_{2 k}} D^{\beta_{1} \ldots \beta_{n-2 k}} F_{\nu \rho}\right) \\
& +\sum_{n=0}^{\infty} \sum_{k=0}^{\lfloor n / 2\rfloor} c_{4}^{(n, k)}\left(\bar{\psi} \gamma_{5} \gamma_{\mu} \stackrel{\leftrightarrow}{D}{ }^{\nu \lambda \alpha_{1} \ldots \alpha_{2 k}} \psi\right)\left(D_{\beta_{1} \ldots \beta_{n-2 k}} F^{\mu \rho} \stackrel{\leftrightarrow}{D}_{\lambda \alpha_{1} \ldots \alpha_{2 k}} D^{\beta_{1} \ldots \beta_{n-2 k}} \tilde{F}_{\nu \rho}\right. \\
& \left.+D_{\beta_{1} \ldots \beta_{n-2 k}} \tilde{F}^{\mu \rho} \stackrel{\leftrightarrow}{D}_{\lambda \alpha_{1} \ldots \alpha_{2 k}} D^{\beta_{1} \ldots \beta_{n-2 k}} F_{\nu \rho}\right)
\end{aligned}
$$

where again we only list the parity-even operators.

\subsection{Operator basis for gravity}

We turn now to the tidal action for gravity. The operator basis for fermions coupled to gravitons is very similar to the electromagnetic case. We have verified that our operator basis produces the helicity amplitudes in table 2 when placed on-shell. 
The full gravitational action includes the minimal coupling for fermions as well as the tidal perturbations to be described:

$$
\sqrt{-g} \mathcal{L}_{\mathrm{GR}}=\sqrt{-g}\left[\bar{\psi}\left(i e^{\mu}{ }_{a} \gamma^{a} D_{\mu}-m\right) \psi+\Delta \mathcal{L}_{\mathrm{GR}}^{\mathrm{odd}}+\Delta \mathcal{L}_{\mathrm{GR}}^{\text {even }}\right]
$$

where the first part of the action is described in detail in e.g. ref. [17]. The tidal contribution at odd mass dimensions is

$$
\begin{aligned}
& \Delta \mathcal{L}_{\mathrm{GR}}^{\mathrm{odd}}=\sum_{n=0}^{\infty} \sum_{k=0}^{\lfloor n / 2\rfloor} d_{1}^{(n, k)}\left(\bar{\psi} \stackrel{\leftrightarrow}{D^{\alpha_{1} \ldots \alpha_{2 k}} \psi}\right)\left(D_{\beta_{1} \ldots \beta_{n-2 k}} C^{\mu \nu \rho \sigma} \stackrel{\leftrightarrow}{D}_{\alpha_{1} \ldots \alpha_{2 k}} D^{\beta_{1} \ldots \beta_{n-2 k}} C_{\mu \nu \rho \sigma}\right) \\
& +\sum_{n=0}^{\infty} \sum_{k=0}^{\lfloor n / 2\rfloor} d_{2}^{(n, k)}\left(\bar{\psi} \stackrel{\leftrightarrow}{D}^{\mu \nu \lambda \tau \alpha_{1} \ldots \alpha_{2 k}} \psi\right)\left(D_{\beta_{1} \ldots \beta_{n-2 k}} C_{\mu \rho \lambda \sigma} \stackrel{\leftrightarrow}{D}_{\alpha_{1} \ldots \alpha_{2 k}} D^{\beta_{1} \ldots \beta_{n-2 k}} C_{\nu \rho \tau \sigma}\right) \\
& +\sum_{n=0}^{\infty} \sum_{k=0}^{\lfloor n / 2\rfloor} i d_{3}^{(n, k)}\left(\bar{\psi} \gamma_{5} \stackrel{\leftrightarrow}{D}^{\mu \nu \lambda \tau \alpha_{1} \ldots \alpha_{2 k+1}} \psi\right)\left(D_{\beta_{1} \ldots \beta_{n-2 k}} C_{\mu \rho \lambda \sigma} \stackrel{\leftrightarrow}{D}_{\alpha_{1} \ldots \alpha_{2 k+1}} D^{\beta_{1} \ldots \beta_{n-2 k}} \tilde{C}_{\nu \rho \tau \sigma}\right)
\end{aligned}
$$

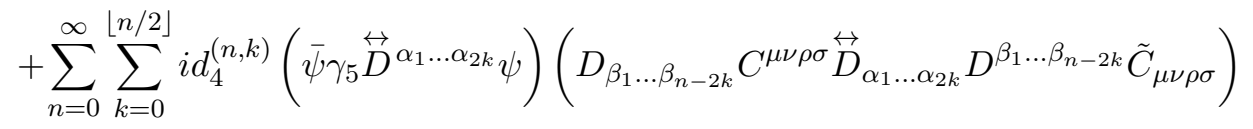

$$
\begin{aligned}
& +\sum_{n=0}^{\infty} i e^{(n)}\left(\bar{\psi} \sigma^{\mu \nu} \stackrel{\leftrightarrow}{D}^{\rho \alpha_{1} \ldots \alpha_{2 n}} \psi\right)\left(C_{\mu \lambda \rho \tau} \stackrel{\leftrightarrow}{D}_{\sigma \alpha_{1} \ldots \alpha_{2 n}} C_{\nu} \lambda \sigma \tau\right)
\end{aligned}
$$

The operators labelled by the Wilson coefficient $e^{(n)}$ produce the gravitational analog of the on-shell structure on the right of eq. (2.9). Again, eq. (2.10) means that these operators only arise at every second odd mass dimension.

The tidal contribution at even mass dimensions is

$$
\begin{aligned}
\Delta \mathcal{L}_{\mathrm{GR}}^{\mathrm{even}}= & \sum_{n=0}^{\infty} \sum_{k=0}^{\lfloor n / 2\rfloor} i f_{1}^{(n, k)}\left(\bar{\psi} \gamma_{\mu} \stackrel{\leftrightarrow}{D} \nu \gamma \delta \alpha_{1} \ldots \alpha_{2 k} \psi\right)\left(D_{\beta_{1} \ldots \beta_{n-2 k}} C^{\mu \rho \gamma \tau} \stackrel{\leftrightarrow}{D}_{\alpha_{1} \ldots \alpha_{2 k}} D^{\beta_{1} \ldots \beta_{n-2 k}} C_{\nu \rho \delta \tau}\right) \\
& +\sum_{n=0}^{\infty} \sum_{k=0}^{\lfloor n / 2\rfloor} i f_{2}^{(n, k)}\left(\bar{\psi} \gamma_{\mu} \stackrel{\leftrightarrow}{D} \nu \gamma \alpha_{1} \ldots \alpha_{2 k} \psi\right)\left(D_{\beta_{1} \ldots \beta_{n-2 k}} C^{\mu \rho \gamma \tau} \stackrel{\leftrightarrow}{D}_{\delta \alpha_{1} \ldots \alpha_{2 k}} D^{\beta_{1} \ldots \beta_{n-2 k}} C_{\nu \rho \delta \tau}\right) \\
& +\sum_{n=0}^{\infty} \sum_{k=0}^{\lfloor n / 2\rfloor} f_{3}^{(n, k)}\left(\bar{\psi} \gamma_{5} \gamma_{\mu} \stackrel{\leftrightarrow}{D} \nu \gamma \delta \lambda \alpha_{1} \ldots \alpha_{2 k} \psi\right)\left(D_{\beta_{1} \ldots \beta_{n-2 k}} C^{\mu \rho \gamma \tau} \stackrel{\leftrightarrow}{D}_{\lambda \alpha_{1} \ldots \alpha_{2 k}} D^{\beta_{1} \ldots \beta_{n-2 k}} \tilde{C}_{\nu \rho \delta \tau}\right. \\
& \left.-D_{\beta_{1} \ldots \beta_{n-2 k}} \tilde{C}^{\mu \rho \gamma \tau} \stackrel{\leftrightarrow}{D}_{\lambda \alpha_{1} \ldots \alpha_{2 k}} D^{\beta_{1} \ldots \beta_{n-2 k}} C_{\nu \rho \delta \tau}\right) \\
& +\sum_{n=0}^{\infty} \sum_{k=0}^{\lfloor n / 2\rfloor} f_{4}^{(n, k)}\left(\bar{\psi} \gamma_{5} \gamma_{\mu} \stackrel{\leftrightarrow}{D}^{\nu \lambda \alpha_{1} \ldots \alpha_{2 k}} \psi\right)\left(D_{\beta_{1} \ldots \beta_{n-2 k}} C^{\mu \rho \gamma \delta} \stackrel{\leftrightarrow}{D}_{\lambda \alpha_{1} \ldots \alpha_{2 k}} D^{\beta_{1} \ldots \beta_{n-2 k}} \tilde{C}_{\nu \rho \gamma \delta}\right. \\
& \left.+D_{\beta_{1} \ldots \beta_{n-2 k}} \tilde{C}^{\mu \rho \gamma \delta} \stackrel{\leftrightarrow}{D}_{\lambda \alpha_{1} \ldots \alpha_{2 k}} D^{\beta_{1} \ldots \beta_{n-2 k}} C_{\nu \rho \gamma \delta}\right)
\end{aligned}
$$

The form of the gravitational action is almost identical to the electromagnetic action. However, note that various operators appear at different mass dimensions compared to the electromagnetic case, due to the additional Lorentz index structure of the Weyl tensors. 


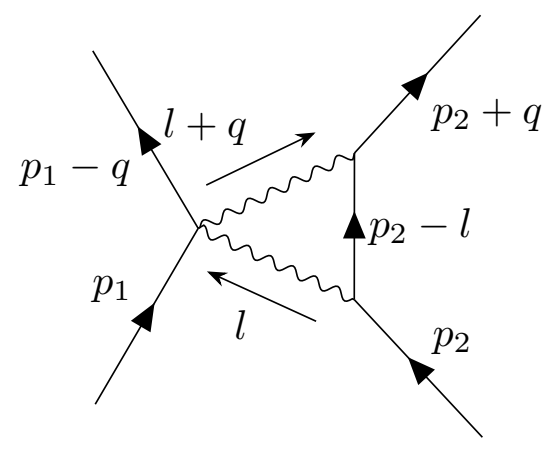

Figure 1. The only topology contributing classical tidal effects at one loop. Here, the tidal effects of particle 1 are probed. The wavy lines represent either photons or gravitons.

\section{$3 \quad$ Leading-PM tidal effects}

The tidal operators listed in eqs. (2.12), (2.13), (2.15) and (2.16) are all we need to compute the leading-PM tidal contributions to spin- $1 / 2-$ spin- $1 / 2$ scattering. There are no contributions at tree-level to the conservative $2 \rightarrow 2$ scattering amplitude, so we must consider the scattering at one loop. The only diagram contributing classically is the triangle diagram, shown in figure 1, where particle 1 is being tidally deformed. Of course, the final result can be symmetrized in the particle labels to obtain the tidal deformation on particle 2 . We let the incoming momenta be $p_{i}^{\mu}=m_{i} v_{i}^{\mu}$, where $v_{i}^{\mu}$ are the particles' four-velocities, which satisfy $v_{i}^{2}=1$. Also, we define $\omega \equiv v_{1} \cdot v_{2}$. As we are interested in the classical portion of the amplitude, we compute the leading-in- $\hbar$ contribution only.

We write the spin effects in terms of the covariant spin vector for heavy particles, defined as

$$
S_{i}^{\mu}=\frac{1}{2} \bar{u}_{v_{i}} \gamma_{5} \gamma^{\mu} u_{v_{i}}
$$

where $\gamma_{5} \equiv-i \gamma^{0} \gamma^{1} \gamma^{2} \gamma^{3}$ and $u_{v_{i}}$ are spinors for a heavy particle with velocity $v_{i}^{\mu}$ - see refs. $[18,59]$ for their relation to Dirac spinors. To do so, we convert all Dirac spinors to heavy spinors at the level of the on-shell amplitudes, and keep only the terms at leading order in $\hbar$. As argued in ref. [18], this spin vector is identifiable with the one-particle matrix element of the spin vector of a classical spinning object: it automatically satisfies the covariant spin supplementary condition (SSC) $p_{i \mu} S^{\mu \nu}=0$ for a spinning object with momentum $p_{i \mu}=m v_{i \mu}$, where $S^{\mu \nu}$ is the classical spin tensor [60]. ${ }^{3}$

The computation of all tidal effects requires knowledge of certain projections of the general-rank triangle integral. Details about these integrals are given in appendix A.

\footnotetext{
${ }^{3}$ The momentum of a spinning object actually deviates from $m v^{\mu}$ by corrections of $\mathcal{O}\left(R S^{2}\right)$, where $R$ is a stand-in for the Riemann tensor [61]. This modifies the SSC at orders cubic in the object's angular momentum. We can safely ignore such effects, as we are focused on contributions at most linear in the spin of either object.
} 


\subsection{QED}

Since we are including spin effects for the tidally deformed particle, as well as for particle 2 , the scattering amplitude will be decomposed in terms of spinless, spin-orbit, and spin-spin contributions. In total, the finite-size contributions at one loop to the QED amplitude for spin- $1 / 2-$ spin- $1 / 2$ scattering are

$$
\begin{aligned}
\Delta \mathcal{A}_{2}^{s=1 / 2}= & \frac{e^{2} S}{\pi^{2}} \sum_{j=0}^{\infty}\left(-\frac{q^{2}}{2}\right)^{j+1}\left[\mathcal{U}_{1} \mathcal{U}_{2} F_{j}^{(0)}-i \omega \mathcal{E}_{1} \mathcal{U}_{2} F_{j}^{(1,1)}+i \omega \mathcal{U}_{1} \mathcal{E}_{2} F_{j}^{(1,2)}\right. \\
& \left.+\left(q \cdot S_{1}\right)\left(q \cdot S_{2}\right) F_{j}^{(2,1)}-q^{2}\left(S_{1} \cdot S_{2}\right) F_{j}^{(2,2)}+\omega q^{2}\left(v_{2} \cdot S_{1}\right)\left(v_{1} \cdot S_{2}\right) F_{j}^{(2,3)}\right]
\end{aligned}
$$

where $e$ is the electromagnetic coupling, $S \equiv \pi^{2} / \sqrt{-q^{2}}$, and $S_{i}^{\mu}$ is the spin vector of particle $i$ defined in eq. (3.1). The subscript 2 indicates that this is the amplitude at quadratic order in the coupling. There are further spin structures that can appear at quadratic order in spin, but they are subleading in the $\hbar$ expansion. The form factors are functions of $\omega$, the dependence on which we leave implicit. We find the form factors to be

$$
\begin{aligned}
F_{j}^{(0)}= & \sum_{k=0}^{j} a_{1}^{(j+k, k)}\left(4 m_{1}\right)^{2 k}\left(1-\omega^{2}\right)^{k} \frac{\alpha_{k}}{4} \\
& +\sum_{k=0}^{j}\left(a_{2}^{(j+k, k)}-\frac{c_{1}^{(j+k, k)}}{2 m_{1}}\right)\left(4 m_{1}\right)^{2 k+2}\left(1-\omega^{2}\right)^{k} \frac{1}{32}\left[\left(\omega^{2}-1\right) \alpha_{k+1}-\omega^{2} \alpha_{k}\right], \\
F_{j}^{(1,1)}= & -\frac{b^{(j)}}{4 m_{2}}\left(4 m_{1}\right)^{2 j}\left(1-\omega^{2}\right)^{j} \alpha_{j} \\
& +\sum_{k=0}^{j} \frac{c_{1}^{(j+k, k)}}{4 m_{1} m_{2}}\left(4 m_{1}\right)^{2 k}\left(1-\omega^{2}\right)^{k}\left[\alpha_{k+1}-\alpha_{k}\right] \\
& +\sum_{k=0}^{j} \frac{c_{4}^{(j+k, k)}-c_{3}^{(j+k, k)}}{8 m_{1} m_{2}}\left(4 m_{1}\right)^{2 k+2}\left(1-\omega^{2}\right)^{k} \alpha_{k+1}, \\
F_{j}^{(1,2)}= & \sum_{k=0}^{j}\left(a_{2}^{(j+k, k)}-\frac{c_{1}^{(j+k, k)}}{2 m_{1}}\right) \frac{\left(4 m_{1}\right)^{2 k+2}}{32 m_{1} m_{2}^{2}}\left(1-\omega^{2}\right)^{k}\left[2 \alpha_{k+1}-\alpha_{k}\right], \\
F_{j}^{(2,1)}= & \sum_{k=0}^{j} \frac{a_{4}^{(j+k, k)}}{4 m_{1} m_{2}}\left(4 m_{1}\right)^{2 k}\left(1-\omega^{2}\right)^{k} \alpha_{k} \\
& +\frac{b^{(j)}}{16 m_{2}}\left(4 m_{1}\right)^{2 j+1}\left(1-\omega^{2}\right)^{j}\left[\left(3 \omega^{2}-2\right)\left(\alpha_{j}+\alpha_{j+1}\right)+\left(2 \omega^{2}-1\right) \frac{2}{2 j+1} \alpha_{j+1}\right] \\
& +\sum_{k=0}^{j} \frac{c_{1}^{(j+k, k)}}{8 m_{2}}\left(4 m_{1}\right)^{2 k}\left(1-\omega^{2}\right)^{k}\left[\left(2 \omega^{2}-1\right)\left(\alpha_{k}-2 \alpha_{k+1}\right)\right] \\
& -\sum_{k=0}^{j} \frac{c_{3}^{(j+k, k)}}{16 m_{2}}\left(4 m_{1}\right)^{2 k+2}\left(1-\omega^{2}\right)^{k+1} \frac{1}{k+2} \alpha_{k+1} \\
& +\sum_{k=0}^{j} \frac{c_{4}^{(j+k, k)}}{8 m_{2}}\left(4 m_{1}\right)^{2 k+2}\left(1-\omega^{2}\right)^{k+1} \alpha_{k+1}, \\
&
\end{aligned}
$$




$$
\begin{aligned}
F_{j}^{(2,2)}= & \frac{b^{(j)}}{16 m_{2}}\left(4 m_{1}\right)^{2 j+1}\left(1-\omega^{2}\right)^{j}\left[\left(2 \omega^{2}-1\right) \frac{1}{2 j+1} \alpha_{j+1}\right] \\
& +\sum_{k=0}^{j} \frac{c_{1}^{(j+k, k)}}{8 m_{2}}\left(4 m_{1}\right)^{2 k}\left(1-\omega^{2}\right)^{k}\left[\left(2 \omega^{2}-1\right)\left(\alpha_{k}-2 \alpha_{k+1}\right)\right] \\
& -\sum_{k=0}^{j} \frac{c_{3}^{(j+k, k)}}{16 m_{2}}\left(4 m_{1}\right)^{2 k+2}\left(1-\omega^{2}\right)^{k+1} \frac{1}{k+2} \alpha_{k+1}, \\
F_{j}^{(2,3)}= & \frac{b^{(j)}}{32 m_{2}}\left(4 m_{1}\right)^{2 j+1}\left(1-\omega^{2}\right)^{j}\left(4 \alpha_{j+1}-\left(2 \omega^{2}-1\right) \alpha_{j+1} \frac{4 j}{2 j+1} \frac{1}{\omega^{2}-1}\right) \\
& +\sum_{k=0}^{j} \frac{c_{1}^{(j+k, k)}}{8 m_{2}}\left(4 m_{1}\right)^{2 k}\left(1-\omega^{2}\right)^{k}\left[\alpha_{k}-\frac{4 k}{2 k+1} \frac{\omega^{2}}{\left(\omega^{2}-1\right)} \alpha_{k+1}\right] \\
& +\sum_{k=0}^{j} \frac{c_{3}^{(j+k, k)}}{4 m_{2}}\left(4 m_{1}\right)^{2 k+2}\left(1-\omega^{2}\right)^{k}\left(\alpha_{k+1}-\frac{2(k+1)}{2 k+3} \alpha_{k+2}\right) .
\end{aligned}
$$

We have introduced the notation

$$
\alpha_{k} \equiv \frac{\left(\frac{1}{2}\right)_{k}}{2^{k}(1)_{k}},
$$

where $(a)_{k}$ is the Pochhammer symbol, and

$$
\begin{aligned}
& u_{v_{1}} \equiv u\left(m_{1} v_{1}\right), \quad u_{v_{2}} \equiv u\left(m_{2} v_{2}\right), \quad \bar{u}_{v_{1}} \equiv \bar{u}\left(m_{1} v_{1}-q\right), \quad \bar{u}_{v_{2}} \equiv \bar{u}\left(m_{2} v_{2}+q\right), \\
& \bar{u}_{v_{1}} u_{v_{1}} \equiv \mathcal{U}_{1}, \quad \bar{u}_{v_{2}} u_{v_{2}} \equiv \mathcal{U}_{2} \\
& v_{2 \mu} q_{\nu} \bar{u}_{v_{1}} \sigma^{\mu \nu} u_{v_{1}}=-2 v_{2 \mu} q_{\nu} \epsilon^{\mu \nu \alpha \beta} v_{1 \alpha} S_{1 \beta} \equiv-2 \mathcal{E}_{1} /\left(m_{1} m_{2}\right) \\
& v_{1 \mu} q_{\nu} \bar{u}_{v_{2}} \sigma^{\mu \nu} u_{v_{2}}=-2 v_{1 \mu} q_{\nu} \epsilon^{\mu \nu \alpha \beta} v_{2 \alpha} S_{2 \beta} \equiv 2 \mathcal{E}_{2} /\left(m_{1} m_{2}\right)
\end{aligned}
$$

to simplify the expressions, where the Levi-Civita tensor is defined by $\epsilon^{0123}=1$. We remark on the absence of contributions from the operators with Wilson coefficients $a_{3}^{(j+k, k)}$ and $c_{2}^{(j+k, k)}$ : the contributions from these operators scale as $\left(q^{2}\right)^{j+2}$, so we treat them as subleading in the $\hbar$ expansion.

Up to a redefinition of Wilson coefficients, the form-factor for the spin-monopole $F_{j}^{(0)}$ agrees with the amplitude in ref. [35]. The matching conditions, accounting for the normalization of the spinors, are

$$
\begin{aligned}
4^{2 k} a_{1}^{(j+k, k)} & \rightarrow \frac{a_{k}^{(j+k)}}{m_{1}}, \\
4^{2 k+2}\left(a_{2}^{(j+k, k)}-\frac{c_{1}^{(j+k, k)}}{2 m_{1}}\right) & \rightarrow-\frac{4}{m_{1}} b_{k}^{(j+k+1)},
\end{aligned}
$$

where the Wilson coefficients on the right hand side are those in ref. [35]. This suggests that the spin-multipole universality for long range electromagnetic scattering [62] extends to the case where tidal deformations are accounted for. This means that, at least at the classical level, the tidally-modified spin-monopole is the same regardless of the total spin of the object. The agreement with the scalar amplitude also shows that the additional even-dimensional operators do not contribute unique spinless structures. 


\subsection{Gravity}

Again, we split the gravitational scattering amplitude in terms of spinless, spin-orbit, and spin-spin contributions. The leading-in- $\hbar$ tidal contributions at $2 \mathrm{PM}$ order are

$$
\begin{aligned}
\Delta \mathcal{M}_{2}^{s=1 / 2}= & G^{2} m_{2}^{2} S \sum_{j=0}^{\infty}\left(-\frac{q^{2}}{2}\right)^{j+2}\left[\mathcal{U}_{1} \mathcal{U}_{2} G_{j}^{(0)}-i \omega \mathcal{E}_{1} \mathcal{U}_{2} G_{j}^{(1,1)}+i \omega \mathcal{U}_{1} \mathcal{E}_{2} G_{j}^{(1,2)}\right. \\
& \left.+\left(q \cdot S_{1}\right)\left(q \cdot S_{2}\right) G_{j}^{(2,1)}-q^{2}\left(S_{1} \cdot S_{2}\right) G_{j}^{(2,2)}+\omega q^{2}\left(v_{2} \cdot S_{1}\right)\left(v_{1} \cdot S_{2}\right) G_{j}^{(2,3)}\right],
\end{aligned}
$$

where the form factors are the following functions of $\omega$ :

$$
\begin{aligned}
& G_{j}^{(0)}=\sum_{k=0}^{j} d_{1}^{(j+k, k)}\left(4 m_{1}\right)^{2 k}\left(1-\omega^{2}\right)^{k} 16 \alpha_{k} \\
& +\sum_{k=0}^{j}\left(d_{2}^{(j+k, k)}-\frac{f_{1}^{(j+k, k)}}{2 m_{1}}\right)\left(4 m_{1}\right)^{2 k+4}\left(1-\omega^{2}\right)^{k} \\
& \times \frac{1}{8}\left[\left(1-2 \omega^{2}\right)^{2} \alpha_{k}+2\left(1-4 \omega^{2}\right)\left(\omega^{2}-1\right) \alpha_{k+1}+2\left(\omega^{2}-1\right)^{2} \alpha_{k+2}\right], \\
& G_{j}^{(1,1)}=-\frac{e^{(j)}}{m_{2}}\left(4 m_{1}\right)^{2 j}\left(1-\omega^{2}\right)^{j} 16 \alpha_{j+1} \\
& +\sum_{k=0}^{j} \frac{f_{1}^{(j+k, k)}}{m_{1} m_{2}}\left(4 m_{1}\right)^{2 k+2}\left(1-\omega^{2}\right)^{k} \\
& \times\left[2\left(\omega^{2}-1\right)\left(\alpha_{k+2}-2 \alpha_{k+1}\right)+\left(2 \omega^{2}-1\right) 2 \alpha_{k}+\left(1-4 \omega^{2}\right) \alpha_{k+1}\right] \\
& +\sum_{k=0}^{j} \frac{f_{3}^{(j+k, k)}}{2 m_{1} m_{2}}\left(4 m_{1}\right)^{2 k+4}\left(1-\omega^{2}\right)^{k}\left[\left(4 \omega^{2}-3\right) \alpha_{k+1}-6\left(\omega^{2}-1\right) \alpha_{k+2}\right] \\
& +\sum_{k=0}^{j} \frac{f_{4}^{(j+k, k)}}{m_{1} m_{2}}\left(4 m_{1}\right)^{2 k+2}\left(1-\omega^{2}\right)^{k} 8 \alpha_{k+1}, \\
& G_{j}^{(1,2)}=\sum_{k=0}^{j}\left(d_{2}^{(j+k, k)}-\frac{f_{1}^{(j+k, k)}}{2 m_{2}}\right) \frac{\left(4 m_{1}\right)^{2 k+4}}{2 m_{1} m_{2}^{2}}\left(1-\omega^{2}\right)^{k} \\
& \times\left[\left(\omega^{2}-1\right)\left(2 \alpha_{k+2}-\alpha_{k+1}\right)-\frac{1}{2}\left(2 \omega^{2}-1\right)\left(2 \alpha_{k+1}-\alpha_{k}\right)\right], \\
& G_{j}^{(2,1)}=\sum_{k=0}^{j} \frac{d_{4}^{(j+k, k)}}{m_{1} m_{2}}\left(4 m_{1}\right)^{2 k}\left(1-\omega^{2}\right)^{k} 16 \alpha_{k} \\
& -\frac{e^{(j)}}{m_{2}}\left(4 m_{1}\right)^{2 j+1}\left(1-\omega^{2}\right)^{j+1} 4 \alpha_{j+1} \\
& -\sum_{k=0}^{j} \frac{f_{1}^{(j+k, k)}}{m_{2}}\left(4 m_{1}\right)^{2 k+2}\left(1-\omega^{2}\right)^{k} \frac{4(2 k+7) \omega^{4}-3(2 k+9) \omega^{2}+3}{8(k+1)(k+2)} \alpha_{k} \\
& +\sum_{k=0}^{j} \frac{f_{3}^{(j+k, k)}}{m_{2}}\left(4 m_{1}\right)^{2 k+4}\left(1-\omega^{2}\right)^{k+1} \frac{3(2 k+7) \omega^{2}-3}{8(k+2)(k+3)} \alpha_{k+1} \\
& +\sum_{k=0}^{j} \frac{f_{4}^{(j+k, k)}}{m_{2}}\left(4 m_{1}\right)^{2 k+2}\left(1-\omega^{2}\right)^{k+1} 8 \alpha_{k+1}
\end{aligned}
$$




$$
\begin{aligned}
G_{j}^{(2,2)}= & -\sum_{k=0}^{j} \frac{f_{1}^{(j+k, k)}}{m_{2}}\left(4 m_{1}\right)^{2 k+2}\left(1-\omega^{2}\right)^{k} \frac{4(2 k+7) \omega^{4}-3(2 k+9) \omega^{2}+3}{8(k+1)(k+2)} \alpha_{k} \\
& +\sum_{k=0}^{j} \frac{f_{3}^{(j+k, k)}}{m_{2}}\left(4 m_{1}\right)^{2 k+4}\left(1-\omega^{2}\right)^{k+1} \frac{3(2 k+7) \omega^{2}-3}{8(k+2)(k+3)} \alpha_{k+1}, \\
G_{j}^{(2,3)}= & \sum_{k=0}^{j} \frac{f_{1}^{(j+k, k)}}{m_{2}}\left(4 m_{1}\right)^{2 k+2}\left(1-\omega^{2}\right)^{k} \\
& \times\left[\frac{\alpha_{k}}{4(k+1)(k+2)\left(\omega^{2}-1\right)}\left(-2(2 k+7) \omega^{4}+(k(2 k+11)+17) \omega^{2}-3(k+1)\right)\right] \\
& +\sum_{k=0}^{j} \frac{f_{3}^{(j+k, k)}}{m_{2}}\left(4 m_{1}\right)^{2 k+4}\left(1-\omega^{2}\right)^{k} \frac{3(k+4)-(k+6)(2 k+7) \omega^{2}}{4(k+2)(k+3)} \alpha_{k+1} .
\end{aligned}
$$

The contributions from the $d_{3}^{(j+k, k)}$ and $f_{2}^{(j+k, k)}$ operators scale as $\left(q^{2}\right)^{j+3}$, so we treat them as subleading in the $\hbar$ expansion.

Results for the amplitude describing the scattering of a tidally defomed, spinless object with a spinning point particle were recently presented in ref. [37]. These are to be compared with the function $G_{j}^{(1,2)}$. After some algebraic manipulations of eq. (3.19) we find agreement with eq. (3.68) in ref. [37] when $k=j$.

As in the electromagnetic case, the form-factor for the spin-monopole $G_{j}^{(0)}$ agrees with the amplitude in ref. [35]; the two sets of Wilson coefficients can be matched through

$$
\begin{aligned}
4^{2 k} d_{1}^{(j+k)} & \rightarrow \frac{c_{k}^{(j+k)}}{m_{1}}, \\
4^{2 k+4}\left(d_{2}^{(j+k, k)}-\frac{f_{1}^{(j+k)}}{2 m_{1}}\right) & \rightarrow \frac{16}{m_{1}} d_{k}^{(j+k+2)},
\end{aligned}
$$

where the coefficients on the right hand side are those in ref. [35]. Under these replacements the form factor here is related to that in ref. [35] through $G_{j}^{(0)} \rightarrow g_{j} / m_{1}$. The differing mass dimensions of the Wilson coefficients in each action is because of the different mass dimensions of scalar versus spinor fields. This matching extends the spin-multipole universality for long range gravitational scattering observed in ref. [63] to the tidally deformed setting. More precisely, this provides evidence that the classical tidally-modified spin-monopole is the same regardless of the total spin of the deformed object.

In both the electromagnetic and gravitational point-particle cases, it is well known that classical spin-spin effects of the form $q^{2} S_{1} \cdot S_{2}$ and $q \cdot S_{1} q \cdot S_{2}$ arise in the proportion $q \cdot S_{1} q \cdot S_{2}-q^{2} S_{1} \cdot S_{2}$ through one-loop order [8, 17,62, 63]. We have shown here that this correlation between spin structures is broken for general values of the Wilson coefficients when finite-size effects are included at the one-loop level.

In the interest of deriving a conservative Hamiltonian in section 4, we rewrite the amplitude in the center-of-mass kinematics of refs. $[8,64]$. These are, for all momenta 
outgoing

$$
\begin{aligned}
& p_{1}^{\mu}=-\left(E_{1}, \boldsymbol{p}\right), \quad p_{2}^{\mu}=-\left(E_{2},-\boldsymbol{p}\right), \quad q^{\mu}=(0, \boldsymbol{q}), \quad \boldsymbol{p} \cdot \boldsymbol{q}=\frac{\boldsymbol{q}^{2}}{2}, \\
& S_{1}^{\mu}=\left(\frac{\boldsymbol{p} \cdot \boldsymbol{S}_{1}}{m_{1}}, \boldsymbol{S}_{1}+\frac{\boldsymbol{p} \cdot \boldsymbol{S}_{1}}{\left(E_{1}+m_{1}\right) m_{1}} \boldsymbol{p}\right), \quad S_{2}^{\mu}=\left(-\frac{\boldsymbol{p} \cdot \boldsymbol{S}_{2}}{m_{2}}, \boldsymbol{S}_{2}+\frac{\boldsymbol{p} \cdot \boldsymbol{S}_{2}}{\left(E_{2}+m_{2}\right) m_{2}} \boldsymbol{p}\right) .
\end{aligned}
$$

Here, $\boldsymbol{S}_{i}$ is the spin vector in the rest frame of particle $i$. It is also the spatial component of the canonical spin vector, which is the spin vector appearing in the canonical Hamiltonian [65]. We express the spin structures that arise using these kinematics: ${ }^{4}$

$$
\begin{aligned}
q \cdot S_{i} & =-\boldsymbol{q} \cdot \boldsymbol{S}_{i}-\frac{\boldsymbol{q}^{2} \boldsymbol{p} \cdot \boldsymbol{S}_{i}}{2 m_{i}\left(E_{i}+m_{i}\right)}, & \mathcal{E}_{i}=E(\boldsymbol{p} \times \boldsymbol{q}) \cdot \boldsymbol{S}_{i}, \\
p_{2} \cdot S_{1} & =-\frac{E}{m_{1}} \boldsymbol{p} \cdot \boldsymbol{S}_{1}, & p_{1} \cdot S_{2}=\frac{E}{m_{2}} \boldsymbol{p} \cdot \boldsymbol{S}_{2}, \\
S_{1} \cdot S_{2} & =-\boldsymbol{S}_{1} \cdot \boldsymbol{S}_{2}-M_{12} \boldsymbol{p} \cdot \boldsymbol{S}_{1} \boldsymbol{p} \cdot \boldsymbol{S}_{2}, &
\end{aligned}
$$

where

$$
M_{12} \equiv \frac{(E+M)^{2}}{2\left(E_{1}+m_{1}\right)\left(E_{2}+m_{2}\right) m_{1} m_{2}},
$$

$E=E_{1}+E_{2}$, and $M=m_{1}+m_{2}$. Substituting these into eq. (3.16) after non-relativistically normalizing the amplitude, and keeping only the leading-in- $\hbar$ terms, we find

$$
\begin{aligned}
\frac{\Delta \mathcal{M}_{2}^{s=1 / 2}}{4 E_{1} E_{2}}= & 2 G^{2} S\left[\mathcal{U}_{1} \mathcal{U}_{2} \Delta a_{\mathrm{cov}, 2}^{(0)}+\Delta a_{\mathrm{cov}, 2}^{(1,1)} i(\boldsymbol{p} \times \boldsymbol{q}) \cdot \boldsymbol{S}_{1} \mathcal{U}_{2}+\Delta a_{\mathrm{cov}, 2}^{(1,2)} \mathcal{U}_{1} i(\boldsymbol{p} \times \boldsymbol{q}) \cdot \boldsymbol{S}_{2}\right. \\
& \left.+\Delta a_{\mathrm{cov}, 2}^{(2,1)}\left(\boldsymbol{q} \cdot \boldsymbol{S}_{1} \boldsymbol{q} \cdot \boldsymbol{S}_{2}\right)+\Delta a_{\mathrm{cov}, 2}^{(2,2)}\left(\boldsymbol{q}^{2} \boldsymbol{S}_{1} \cdot \boldsymbol{S}_{2}\right)+\Delta a_{\mathrm{cov}, 2}^{(2,3)} \boldsymbol{q}^{2} \boldsymbol{p} \cdot \boldsymbol{S}_{1} \boldsymbol{p} \cdot \boldsymbol{S}_{2}\right],
\end{aligned}
$$

where

$$
\begin{aligned}
& \Delta a_{\mathrm{cov}, 2}^{(0)}=m_{2}^{2} \sum_{j=0}^{\infty}\left(\frac{\boldsymbol{q}^{2}}{2}\right)^{j+2}\left[\frac{G_{j}^{(0)}}{8 E_{1} E_{2}}\right], \\
& \Delta a_{\mathrm{cov}, 2}^{(1,1)}=m_{2}^{2} \sum_{j=0}^{\infty}\left(\frac{\boldsymbol{q}^{2}}{2}\right)^{j+2}\left[-\frac{\omega E}{8 E_{1} E_{2}} G_{j}^{(1,1)}\right], \\
& \Delta a_{\mathrm{cov}, 2}^{(1,2)}=m_{2}^{2} \sum_{j=0}^{\infty}\left(\frac{\boldsymbol{q}^{2}}{2}\right)^{j+2}\left[\frac{\omega E}{8 E_{1} E_{2}} G_{j}^{(1,2)}\right], \\
& \Delta a_{\mathrm{cov}, 2}^{(2,1)}=m_{2}^{2} \sum_{j=0}^{\infty}\left(\frac{\boldsymbol{q}^{2}}{2}\right)^{j+2}\left[\frac{1}{8 E_{1} E_{2}} G_{j}^{(2,1)}\right] \\
& \Delta a_{\mathrm{cov}, 2}^{(2,2)}=m_{2}^{2} \sum_{j=0}^{\infty}\left(\frac{\boldsymbol{q}^{2}}{2}\right)^{j+2}\left[-\frac{1}{8 E_{1} E_{2}} G_{j}^{(2,2)}\right], \\
& \Delta a_{\mathrm{cov}, 2}^{(2,3)}=m_{2}^{2} \sum_{j=0}^{\infty}\left(\frac{\boldsymbol{q}^{2}}{2}\right)^{j+2}\left[\frac{\omega E^{2}}{8 E_{1} E_{2} m_{1}^{2} m_{2}^{2}} G_{j}^{(2,3)}-\frac{M_{12}}{8 E_{1} E_{2}} G_{j}^{(2,2)}\right] .
\end{aligned}
$$

\footnotetext{
${ }^{4}$ We use the mostly negative metric signature. Note that we find the opposite sign on the first term of $q \cdot S_{i}$ relative to ref. [8]. This difference is immaterial, however, since these structures only arise in the combination $q \cdot S_{1} q \cdot S_{2}$, causing the signs to cancel, and since the second term in this inner product is subleading in $\hbar$.
} 
We have borrowed the notation from ref. [8], where the subscript cov denotes that these are the coefficients to the spin structures when the amplitude is written in terms of the covariant spin vectors. We define the notation

$$
\Delta a_{\mathrm{cov}, 2}^{A} \equiv m_{2}^{2} \sum_{j=0}^{\infty}\left(\frac{\boldsymbol{q}^{2}}{2}\right)^{j+2} \Delta a_{\mathrm{cov}, 2, j}^{A}(\omega),
$$

for easy reference later on.

\section{Conservative two-body Hamiltonian}

In this section, we use the effective field theory (EFT) matching approach of ref. [8] to derive the two-body spin-dependent conservative Hamiltonian. Working with the spin-coherent states $|\boldsymbol{n}\rangle$ defined therein, the two-body Hamiltonian is given by

$$
H(\boldsymbol{q}, \boldsymbol{p})=\sqrt{\boldsymbol{p}^{2}+m_{1}^{2}}+\sqrt{\boldsymbol{p}^{2}+m_{2}^{2}}+\left\langle\boldsymbol{n}_{1} \boldsymbol{n}_{2}\left|\left[\hat{V}\left(\boldsymbol{k}^{\prime}, \boldsymbol{k}, \hat{\boldsymbol{S}}_{a}\right)+\Delta \hat{V}\left(\boldsymbol{k}^{\prime}, \boldsymbol{k}, \hat{\boldsymbol{S}}_{a}\right)\right]\right| \boldsymbol{n}_{1} \boldsymbol{n}_{2}\right\rangle,
$$

where $\Delta \hat{V}$ encodes the tidal contributions to the Hamiltonian for spinning particles. Here, $\boldsymbol{k}$ is the incoming three-momentum, $\boldsymbol{k}^{\prime}$ is the outgoing three-momentum, $\boldsymbol{q} \equiv \boldsymbol{k}-\boldsymbol{k}^{\prime}$ is the transferred three-momentum, and $\boldsymbol{p} \equiv\left(\boldsymbol{k}^{\prime}+\boldsymbol{k}\right) / 2$. Finally, $\hat{\boldsymbol{S}}_{a}$ is the rest-frame spin operator of particle $a$, whose expectation value in the spin-coherent state of particle $a$ gives its rest-frame spin vector, $\boldsymbol{S}_{a}$. The tidal potential can be expanded in the basis of spin operators analogously to the expansion of $\hat{V}\left(\boldsymbol{k}^{\prime}, \boldsymbol{k}, \hat{\boldsymbol{S}}_{a}\right)$ in ref. [8]:

$$
\Delta \hat{V}\left(\boldsymbol{k}^{\prime}, \boldsymbol{k}, \hat{\boldsymbol{S}}_{a}\right)=\sum_{A} \Delta \hat{V}^{A}\left(\boldsymbol{k}^{\prime}, \boldsymbol{k}\right) \hat{\mathbb{O}}^{A} .
$$

$A$ labels the following classical spin structures:

$$
\begin{aligned}
& \hat{\mathbb{O}}^{(0)}=\mathbb{I}, \quad \hat{\mathbb{O}}^{(1,1)}=\boldsymbol{L}_{q} \cdot \hat{\boldsymbol{S}}_{1}, \quad \hat{\mathbb{O}}^{(1,2)}=\boldsymbol{L}_{q} \cdot \hat{\boldsymbol{S}}_{2}, \\
& \hat{\mathbb{O}}^{(2,1)}=\boldsymbol{q} \cdot \hat{\boldsymbol{S}}_{1} \boldsymbol{q} \cdot \hat{\boldsymbol{S}}_{2}, \quad \hat{\mathbb{O}}^{(2,2)}=\boldsymbol{q}^{2} \hat{\boldsymbol{S}}_{1} \cdot \hat{\boldsymbol{S}}_{2}, \quad \hat{\mathbb{O}}^{(2,3)}=\boldsymbol{q}^{2} \boldsymbol{p} \cdot \hat{\boldsymbol{S}}_{1} \boldsymbol{p} \cdot \hat{\boldsymbol{S}}_{2},
\end{aligned}
$$

where $\boldsymbol{L}_{q} \equiv i(\boldsymbol{p} \times \boldsymbol{q})$. The first index in the superscripts labels the number of spin vectors in the operator, whereas the second labels the different structures with that many spin vectors. There are two spin structures that are not included in this basis. They are

$$
\boldsymbol{q} \cdot \boldsymbol{p} \boldsymbol{q} \cdot \hat{\boldsymbol{S}}_{1} \boldsymbol{p} \cdot \hat{\boldsymbol{S}}_{2}, \quad \boldsymbol{q} \cdot \boldsymbol{p} \boldsymbol{p} \cdot \hat{\boldsymbol{S}}_{1} \boldsymbol{q} \cdot \hat{\boldsymbol{S}}_{2}
$$

As discussed in ref. [8], these are omitted from the basis since the on-shell condition $\boldsymbol{q} \cdot \boldsymbol{p} \sim \boldsymbol{q}^{2}$ means they are subleading in the $\hbar$ expansion.

To match the tidal amplitude to the tidal potential, the coefficients of these operators are also expanded in powers of $G$ :

$$
\Delta \hat{V}^{A}\left(\boldsymbol{k}^{\prime}, \boldsymbol{k}\right)=\frac{4 \pi G}{\boldsymbol{q}^{2}} \Delta d_{1}^{A}\left(\boldsymbol{k}^{\prime}, \boldsymbol{k}\right)+\frac{2 \pi^{2} G^{2}}{|\boldsymbol{q}|} \Delta d_{2}^{A}\left(\boldsymbol{k}^{\prime}, \boldsymbol{k}\right)+\mathcal{O}\left(G^{3}\right) .
$$

Tidal effects arise first at $\mathcal{O}\left(G^{2}\right)$, which imposes $\Delta d_{1}^{A}\left(\boldsymbol{k}^{\prime}, \boldsymbol{k}\right)=0$. Note also that the tidal effects allow for higher powers of $\boldsymbol{q}^{2}$ to contribute classically to the potential at a given 


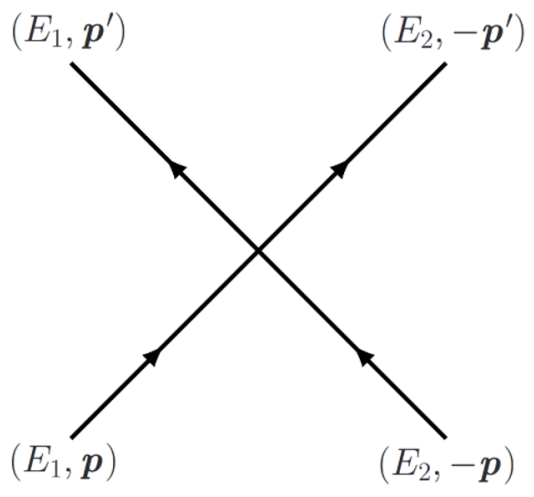

Figure 2. The diagram encoding the leading tidal effect in the EFT in eq. (4.6).

order in $G$. This potential is computed from the EFT of ref. [8], whose action for spin- $1 / 2$ fermions is given by

$$
\begin{aligned}
S=\int_{\boldsymbol{k}} \sum_{a=1,2} \psi_{a}^{\dagger} & -\boldsymbol{k})\left(i \partial_{t}-\sqrt{\boldsymbol{k}^{2}+m_{i}^{2}}\right) \psi_{a}(\boldsymbol{k}) \\
& -\int_{\boldsymbol{k}, \boldsymbol{k}^{\prime}} \psi_{1}^{\dagger}\left(\boldsymbol{k}^{\prime}\right) \psi_{2}^{\dagger}\left(-\boldsymbol{k}^{\prime}\right)\left(\hat{V}\left(\boldsymbol{k}^{\prime}, \boldsymbol{k}, \hat{\boldsymbol{S}}_{a}\right)+\Delta \hat{V}\left(\boldsymbol{k}^{\prime}, \boldsymbol{k}, \hat{\boldsymbol{S}}_{a}\right)\right) \psi_{1}(\boldsymbol{k}) \psi_{2}(-\boldsymbol{k}) .
\end{aligned}
$$

The coefficients $\Delta d_{i}^{A}\left(\boldsymbol{k}^{\prime}, \boldsymbol{k}\right)$ are then found by matching the amplitudes from the above EFT with the amplitudes derived from the tidal actions given in sections 2.4 and 2.5.

We move now to the computation of the tidal amplitude from this EFT. As noted above, $\Delta d_{1}^{A}\left(\boldsymbol{k}^{\prime}, \boldsymbol{k}\right)=0$, which means the leading tidal potential is an $\mathcal{O}\left(G^{2}\right)$ quantity. As we wish to match to the $\mathcal{O}\left(G^{2}\right)$ amplitude from the full theory, this means that we will only need to compute the tidal contribution to the EFT amplitude from the tree-level diagram in figure 2. This amplitude is simply

$$
\Delta \mathcal{M}_{2}^{\mathrm{EFT}}=-\left\langle\boldsymbol{n}_{1} \boldsymbol{n}_{2}\left|\Delta \hat{V}\left(\boldsymbol{k}^{\prime}, \boldsymbol{k}, \hat{\boldsymbol{S}}_{i}\right)\right| \boldsymbol{n}_{1} \boldsymbol{n}_{2}\right\rangle \equiv-\Delta V\left(\boldsymbol{k}^{\prime}, \boldsymbol{k}, \boldsymbol{S}_{i}\right)
$$

The subscript 2 on the left-hand side reminds us that this is an amplitude at $\mathcal{O}\left(G^{2}\right)$. We can also expand the EFT amplitude in the basis of eq. (4.3):

$$
\begin{aligned}
\Delta \mathcal{M}_{2}^{\mathrm{EFT}}=\frac{2 \pi^{2} G^{2}}{|\boldsymbol{q}|}[ & \Delta a_{2}^{(0)}+\Delta a_{2}^{(1,1)} \boldsymbol{L}_{q} \cdot \boldsymbol{S}_{1}+a_{2}^{(1,2)} \boldsymbol{L}_{q} \cdot \boldsymbol{S}_{2} \\
& \left.+\Delta a_{2}^{(2,1)} \boldsymbol{q} \cdot \boldsymbol{S}_{1} \boldsymbol{q} \cdot \boldsymbol{S}_{2}+\Delta a_{2}^{(2,2)} \boldsymbol{q}^{2} \boldsymbol{S}_{1} \cdot \boldsymbol{S}_{2}+\Delta a_{2}^{(2,3)} \boldsymbol{q} \boldsymbol{p} \cdot \boldsymbol{S}_{1} \boldsymbol{p} \cdot \boldsymbol{S}_{2}\right]
\end{aligned}
$$

Combining this with eqs. (4.2), (4.5) and (4.7) and matching the coefficients on the different spin operators, we arrive at the simple relation to leading-PM order in tidal effects:

$$
\Delta a_{2}^{A}=-\Delta d_{2}^{A}
$$

Now, the EFT in eq. (4.6) is the effective theory where the graviton modes have been integrated out of the full theory. As an effective version of the full theory, it must produce 
the same low-energy amplitudes as the full theory:

$$
\Delta \mathcal{M}_{2}^{\mathrm{EFT}}=\frac{\Delta \mathcal{M}_{2}^{s=1 / 2}}{4 E_{1} E_{2}}
$$

where we have non-relativistically normalized the full theory amplitude. Note that since both of these amplitudes are the leading order where tidal effects arise, there are no iteration terms to be matched. Before we can match these two amplitudes, we must first express the spinor products in the amplitude in eq. (3.28) in terms of the canonical spin vector. The result of this is the mixing of the $\Delta a_{\mathrm{cov}, 2}^{A}$ coefficients, which has been worked out in ref. [8]. ${ }^{5}$ The mixing derived there is slightly modified in our case, as our spinors are dimensionful. Accounting for this mixing, eq. (4.10) yields the matching conditions

$$
\begin{aligned}
\Delta a_{2}^{(0)} & =4 m_{1} m_{2} \Delta a_{\mathrm{cov}, 2}^{(0)}, \\
\Delta a_{2}^{(1,1)} & =2 m_{2} \Delta a_{\mathrm{cov}, 2}^{(1,1)}-\frac{4 m_{2} \Delta a_{\mathrm{cov}, 2}^{(0)}}{E\left(p_{1}\right)+m_{1}}, \\
\Delta a_{2}^{(1,2)} & =2 m_{1} \Delta a_{\mathrm{cov}, 2}^{(1,2)}-\frac{4 m_{1} \Delta a_{\mathrm{cov}, 2}^{(0)}}{E\left(p_{2}\right)+m_{2}} \\
\Delta a_{2}^{(2,1)} & =\Delta a_{\mathrm{cov}, 2}^{(2,1)}-\frac{2 \boldsymbol{p}^{2} \Delta a_{\mathrm{cov}, 2}^{(1,1)}}{E\left(p_{2}\right)+m_{2}}-\frac{2 \boldsymbol{p}^{2} \Delta a_{\mathrm{cov}, 2}^{(1,2)}}{E\left(p_{1}\right)+m_{1}}+\frac{4 \boldsymbol{p}^{2} \Delta a_{\mathrm{cov}, 2}^{(0)}}{\left[E\left(p_{1}\right)+m_{1}\right]\left[E\left(p_{2}\right)+m_{2}\right]}, \\
\Delta a_{2}^{(2,2)} & =\Delta a_{\mathrm{cov}, 2}^{(2,1)}+\frac{2 \boldsymbol{p}^{2} \Delta a_{\mathrm{cov}, 2}^{(1,1)}}{E\left(p_{2}\right)+m_{2}}+\frac{2 \boldsymbol{p}^{2} \Delta a_{\mathrm{cov}, 2}^{(1,2)}}{E\left(p_{1}\right)+m_{1}}-\frac{4 \boldsymbol{p}^{2} \Delta a_{\mathrm{cov}, 2}^{(0)}}{\left[E\left(p_{1}\right)+m_{1}\right]\left[E\left(p_{2}\right)+m_{2}\right]}, \\
\Delta a_{2}^{(2,3)} & =\Delta a_{\mathrm{cov}, 2}^{(2,1)}-\frac{2 \Delta a_{\mathrm{cov}, 2}^{(1,1)}}{E\left(p_{2}\right)+m_{2}}-\frac{2 \Delta a_{\mathrm{cov}, 2}^{(1,2)}}{E\left(p_{1}\right)+m_{1}}+\frac{4 \Delta a_{\mathrm{cov}, 2}^{(0)}}{\left[E\left(p_{1}\right)+m_{1}\right]\left[E\left(p_{2}\right)+m_{2}\right]} .
\end{aligned}
$$

Combining this with eqs. (4.7) to (4.9), the momentum-space potential at $\mathcal{O}\left(G^{2}\right)$ can be written as

$$
\Delta V\left(\boldsymbol{k}^{\prime}, \boldsymbol{k}, \boldsymbol{S}_{a}\right)=-\frac{2 \pi^{2} m_{2}^{2} G^{2}}{|\boldsymbol{q}|} \sum_{j=0}^{\infty}\left(\frac{\boldsymbol{q}^{2}}{2}\right)^{j+2} \sum_{A} \Delta a_{2, j}^{A}(\omega) \mathbb{O}^{A}
$$

where the $\Delta a_{2, j}^{A}(\omega)$ are defined through

$$
\Delta a_{2}^{A}(\omega) \equiv m_{2}^{2} \sum_{j=0}^{\infty}\left(\frac{\boldsymbol{q}^{2}}{2}\right)^{j+2} \Delta a_{2, j}^{A}(\omega)
$$

The potential can be expressed in position space by simply Fourier transforming, giving

\footnotetext{
${ }^{5}$ We thank Andrés Luna for discussion on this point.
} 
(see appendix B for the relevant integrals)

$$
\begin{aligned}
\Delta V\left(\boldsymbol{r}, \boldsymbol{p}, \boldsymbol{S}_{a}\right)= & -\frac{\sqrt{\pi} m_{2}^{2} G^{2}}{2} \sum_{j=0}^{\infty}\left(\frac{2}{\boldsymbol{r}^{2}}\right)^{j+3} \Gamma(j+3)\left[\frac{1}{\Gamma(-j-3 / 2)} \Delta a_{2, j}^{(0)}(\omega) \mathbb{I}\right. \\
& -\left(\frac{2}{\boldsymbol{r}^{2}}\right) \frac{j+3}{\Gamma(-j-3 / 2)}\left(\Delta a_{2, j}^{(1,1)}(\omega) \boldsymbol{L} \cdot \boldsymbol{S}_{1}+\Delta a_{2, j}^{(1,2)}(\omega) \boldsymbol{L} \cdot \boldsymbol{S}_{2}\right) \\
& -2(j+4)\left(\frac{2}{\boldsymbol{r}^{2}}\right) \frac{1}{\boldsymbol{r}^{2}} \frac{j+3}{\Gamma(-j-3 / 2)} \Delta a_{2, j}^{(2,1)}(\omega) \boldsymbol{r} \cdot \boldsymbol{S}_{1} \boldsymbol{r} \cdot \boldsymbol{S}_{2} \\
& +\left(\frac{2}{\boldsymbol{r}^{2}}\right)(j+3)\left(\frac{1}{\Gamma(-j-3 / 2)} \Delta a_{2, j}^{(2,1)}(\omega)+\frac{2}{\Gamma(-j-5 / 2)} \Delta a_{2, j}^{(2,2)}(\omega)\right) \boldsymbol{S}_{1} \cdot \boldsymbol{S}_{2} \\
& \left.+2\left(\frac{2}{\boldsymbol{r}^{2}}\right) \frac{j+3}{\Gamma(-j-5 / 2)} \Delta a_{2, j}^{(2,3)}(\omega) \boldsymbol{p} \cdot \boldsymbol{S}_{1} \boldsymbol{p} \cdot \boldsymbol{S}_{2}\right],
\end{aligned}
$$

where $\boldsymbol{L} \equiv \boldsymbol{r} \times \boldsymbol{p}$ is the angular momentum. The spin-monopole portion of this potential agrees with that found in ref. [35] after performing the matching in eqs. (3.23) and (3.24)

\section{Classical observables}

The scattering amplitude can be related to various classical observables. For example, refs. $[4,6]$ derived direct relations between the scattering amplitude and the linear and angular impulses. In this section, we will use the tidally deformed scattering amplitudes for spin-1/2 particles, eqs. (3.2) and (3.16), to find the tidal contributions to these quantities. We remark that the $\Delta$ prefixes in the remainder of this section denote changes in the linear and angular momenta.

\subsection{Linear impulse}

Following ref. [4], the leading term for the classical linear impulse is ${ }^{6}$

$$
\Delta p_{1}^{\mu}=\left\langle\left\langle i \int \hat{d}^{4} q \hat{\delta}\left(2 p_{1} \cdot q\right) \hat{\delta}\left(2 p_{2} \cdot q\right) e^{-i b \cdot q} q^{\mu} \mathcal{A}\left(p_{1}, p_{2} \rightarrow p_{1}+q, p_{2}-q\right)\right\rangle\right\rangle,
$$

where $b^{\mu}$ is the impact parameter. Since we are dealing with the leading-PM tidal amplitudes, we do not need to include the second contribution to the linear impulse, i.e. eq. (3.30) in ref. [4]. The effect of the double angle brackets is to take the expectation values for the spins, as well as to impose the replacement $p_{i} \rightarrow m_{i} v_{i}$ on the initial momenta. As we have already written the amplitudes by expressing the momenta in this way, the task is reduced to the computation of integrals of the form in eq. (B.5). We present all computational details in appendix $\mathrm{B}$ and write here just the results.

Before computing the impulse, we highlight that we will work with the covariant spin vector in this section. This changes the way the spinor products $\mathcal{U}_{i}$ are treated compared to section 4. First, note that the spinors in the amplitudes of eqs. (3.2) and (3.16) are normalized such that $\bar{u}(p) u(p)=2 m$. Moreover, as the momenta of the final states differ

\footnotetext{
${ }^{6}$ We leave the factors of $\hbar$ implicit.
} 
from those of the initial states by momenta of order $\hbar$, we can expand the final state spinors in powers of $\hbar$ :

$$
\bar{u}(p \pm \hbar \bar{q})=\bar{u}(p)+\mathcal{O}(q) .
$$

In fact, when working with the covariant spin vector, this $\mathcal{O}(q)$ correction is an infinitesimal Lorentz boost of the spinor [6]. This applies just as well to the heavy spinors, so, to leading order in $q$, the spinor products are $\mathcal{U}_{i}=2 m_{i}+\mathcal{O}\left(q^{2}\right)$.

We begin with the linear impulse in the gravitational case. Plugging the tidal contribution to the amplitude, eq. (3.16), into the impulse formula we have that ${ }^{7}$

$$
\begin{aligned}
\Delta p_{1, \mathrm{GR}}^{\mu}= & i G^{2} m_{2}^{2} \sum_{j=0}^{\infty}\left[4 m_{1} m_{2} G_{j}^{(0)} I_{j+2}^{\mu}(-b)\right. \\
& -2 i \omega m_{1} m_{2} \epsilon_{\rho \nu \alpha \beta} v_{1}^{\nu} v_{2}^{\alpha}\left(m_{2}\left\langle S_{1}^{\beta}\right\rangle G_{j}^{(1,1)}-m_{1}\left\langle S_{2}^{\beta}\right\rangle G_{j}^{(1,2)}\right) I_{j+2}^{\mu \rho}(-b) \\
& \left.+\left(\left\langle S_{1 \nu}\right\rangle\left\langle S_{2 \rho}\right\rangle G_{j}^{(2,1)}-\eta_{\nu \rho}\left\langle S_{1}\right\rangle \cdot\left\langle S_{2}\right\rangle G_{j}^{(2,2)}+\omega \eta_{\nu \rho}\left(v_{2} \cdot\left\langle S_{1}\right\rangle\right)\left(v_{1} \cdot\left\langle S_{2}\right\rangle\right) G_{j}^{(2,3)}\right) I_{j+2}^{\mu \nu \rho}(-b)\right] .
\end{aligned}
$$

By using the results for the integrals in eqs. (B.8), (B.13) and (B.17), we end up with

$$
\begin{aligned}
\Delta p_{1, \mathrm{GR}}^{\mu}= & \frac{-\pi G^{2} m_{2}}{8 m_{1} \sqrt{\omega^{2}-1}} \sum_{j=0}^{\infty}\left(-\frac{2}{b^{2}}\right)^{j+3} \frac{\Gamma[7 / 2+j]}{\Gamma[-3 / 2-j]}\left[-4 m_{1} m_{2} G_{j}^{(0)} \frac{b^{\mu}}{|\boldsymbol{b}|}\right. \\
& +2 \omega m_{1} m_{2}^{2} \epsilon_{\rho \nu \alpha \beta} v_{2}^{\nu} v_{1}^{\alpha}\left\langle S_{1}^{\beta}\right\rangle G_{j}^{(1,1)} \frac{1}{|\boldsymbol{b}|}\left((7+2 j) \frac{b^{\mu} b^{\rho}}{b^{2}}-\Pi^{\mu \rho}\right) \\
& +2 \omega m_{1}^{2} m_{2} \epsilon_{\rho \nu \alpha \beta} v_{1}^{\nu} v_{2}^{\alpha}\left\langle S_{2}^{\beta}\right\rangle G_{j}^{(1,2)} \frac{1}{|\boldsymbol{b}|}\left((7+2 j) \frac{b^{\mu} b^{\rho}}{b^{2}}-\Pi^{\mu \rho}\right) \\
& -\left[\left\langle S_{1 \nu}\right\rangle\left\langle S_{2 \rho}\right\rangle G_{j}^{(2,1)}-\eta_{\nu \rho}\left(\left\langle S_{1}\right\rangle \cdot\left\langle S_{2}\right\rangle\right) G_{j}^{(2,2)}+\omega \eta_{\nu \rho}\left(v_{2} \cdot\left\langle S_{1}\right\rangle\right)\left(v_{1} \cdot\left\langle S_{2}\right\rangle\right) G_{j}^{(2,3)}\right] \\
& \left.\times\left((9 / 2+j) \frac{b^{\mu} b^{\nu} b^{\rho}}{b^{2}}-\frac{3}{2} b^{(\mu} \Pi^{\nu \rho)}\right) \frac{2}{|\boldsymbol{b}|}\left(-\frac{2}{b^{2}}\right)(7 / 2+j)\right],
\end{aligned}
$$

where $\Pi^{\mu \nu}$ is defined in eq. (B.10). In the electromagnetic case, the impulse is

$$
\begin{aligned}
\Delta p_{1, \mathrm{EM}}^{\mu}= & \frac{-e^{2}}{8 \pi m_{1} m_{2} \sqrt{\omega^{2}-1}} \sum_{j=0}^{\infty}\left(-\frac{2}{b^{2}}\right)^{j+2} \frac{\Gamma[5 / 2+j]}{\Gamma[-1 / 2-j]}\left[-4 m_{1} m_{2} F_{j}^{(0)} \frac{b^{\mu}}{|\boldsymbol{b}|}\right. \\
& +2 \omega m_{1} m_{2}^{2} \epsilon_{\rho \nu \alpha \beta} v_{2}^{\nu} v_{1}^{\alpha}\left\langle S_{1}^{\beta}\right\rangle F_{j}^{(1,1)} \frac{1}{|\boldsymbol{b}|}\left((5+2 j) \frac{b^{\mu} b^{\rho}}{b^{2}}-\Pi^{\mu \rho}\right) \\
& +2 \omega m_{1}^{2} m_{2} \epsilon_{\rho \nu \alpha \beta} v_{1}^{\nu} v_{2}^{\alpha}\left\langle S_{2}^{\beta}\right\rangle F_{j}^{(1,2)} \frac{1}{|\boldsymbol{b}|}\left((5+2 j) \frac{b^{\mu} b^{\rho}}{b^{2}}-\Pi^{\mu \rho}\right) \\
& -\left[\left\langle S_{1 \nu}\right\rangle\left\langle S_{2 \rho}\right\rangle F_{j}^{(2,1)}-\eta_{\nu \rho}\left(\left\langle S_{1}\right\rangle \cdot\left\langle S_{2}\right\rangle\right) F_{j}^{(2,2)}+\omega \eta_{\nu \rho}\left(v_{2} \cdot\left\langle S_{1}\right\rangle\right)\left(v_{1} \cdot\left\langle S_{2}\right\rangle\right) F_{j}^{(2,3)}\right] \\
& \left.\times\left((7 / 2+j) \frac{b^{\mu} b^{\nu} b^{\rho}}{b^{2}}-\frac{3}{2} b^{(\mu} \Pi^{\nu \rho)}\right) \frac{2}{|\boldsymbol{b}|}\left(-\frac{2}{b^{2}}\right)(5 / 2+j)\right] .
\end{aligned}
$$

\footnotetext{
${ }^{7}$ Note that eq. (5.1) gives the impulse for the particle that absorbs the transfer momentum. In our case, particle 1 is the emitting particle, so we must evaluate the integrals at $-b^{\mu}$.
} 
Note that the electromagnetic result can be obtained from the gravitational result using the following replacements:

$$
\begin{aligned}
G^{2} m_{2}^{2} & \rightarrow e^{2} / \pi^{2}, \\
G_{j}^{A} & \rightarrow F_{j}^{A}, \\
j & \rightarrow j-1 .
\end{aligned}
$$

The last of these replacements is not applied to the indices of the form factors.

\subsection{Angular impulse}

We turn now to the determination of the angular impulse. The angular impulse for the absorbing particle is related to the amplitude through $[6]^{8}$

$$
\Delta S_{1}^{\mu}=\left\langle\left\langle i \int \hat{d}^{4} q \hat{\delta}\left(2 p_{1} \cdot q\right) \hat{\delta}\left(2 p_{2} \cdot q\right) e^{-i b \cdot q}\left(-\frac{p_{1}^{\mu}}{m_{1}^{2}} q \cdot S_{1}\left(p_{1}\right) \mathcal{A}(q)+\left[S_{1}^{\mu}\left(p_{1}\right), \mathcal{A}(q)\right]\right)\right\rangle\right\rangle,
$$

where $p_{1}^{\mu}$ is the initial momentum of the absorbing particle. We don't need the second contribution to the impulse, eq. (3.22) in ref. [6], because we are calculating the leadingPM tidal contribution. For the spin-1/2 amplitudes we are considering, this formula will produce terms of $\mathcal{O}\left(S_{i}^{2}\right)$. We ignore such contributions since one must consider spin-1 scattering to obtain all information at this spin order.

We compute each term individually for particle 1, which is the emitting particle in our setup. The first term is

$$
\left\langle\left\langle i \int \hat{d}^{4} q \hat{\delta}\left(2 p_{1} \cdot q\right) \hat{\delta}\left(2 p_{2} \cdot q\right) e^{i b \cdot q} \frac{p_{1}^{\mu}}{m_{1}^{2}} q \cdot S_{1}\left(p_{1}\right) \mathcal{A}_{2}(q)\right\rangle\right\rangle=-\frac{v_{1}^{\mu}}{m_{1}} \Delta p_{1} \cdot\left\langle S_{1}\right\rangle
$$

which follows since, to the order we are working, there is only one contribution to the linear impulse. This is true for both the electromagnetic and gravitational cases. Writing this explicitly for the case of gravity,

$$
\begin{aligned}
& -\frac{v_{1}^{\mu}}{m_{1}} \Delta p_{1} \cdot\left\langle S_{1}\right\rangle=\frac{v_{1}^{\mu} \pi G^{2} m_{2}^{2}}{4 m_{1}|\mathbf{b}| \sqrt{\omega^{2}-1}} \sum_{j=0}^{\infty}\left(-\frac{2}{b^{2}}\right)^{j+3} \frac{\Gamma[7 / 2+j]}{\Gamma[-3 / 2-j]} \\
& \times\left[-2 G_{j}^{(0)} b^{\lambda}+\omega m_{1} \epsilon_{\rho \nu \alpha \beta} v_{1}^{\nu} v_{2}^{\alpha}\left\langle S_{2}^{\beta}\right\rangle G_{j}^{(1,2)}\left((7+2 j) \frac{b^{\lambda} b^{\rho}}{b^{2}}-\Pi^{\lambda \rho}\right)\right]\left\langle S_{1 \lambda}\right\rangle+\mathcal{O}\left(\left\langle S_{1}\right\rangle^{2}\right) .
\end{aligned}
$$

To compute the commutator term, we need the following commutator [6]:

$$
\left[S_{i}^{\mu}, S_{j}^{\nu}\right]=-\delta_{i j} \frac{i}{m_{i}} \epsilon^{\mu \nu \rho \sigma} S_{i \rho} p_{i \sigma}
$$

\footnotetext{
${ }^{8}$ Again, we leave the factors of $\hbar$ implicit.
} 
With this in hand, the commutator term for gravity is

$$
\begin{aligned}
& \left\langle\left\langle i \int \hat{d}^{4} q \hat{\delta}\left(2 p_{1} \cdot q\right) \hat{\delta}\left(2 p_{2} \cdot q\right) e^{i b \cdot q}\left[S_{1}^{\mu}\left(p_{1}\right), \mathcal{M}_{2}(q)\right]\right\rangle\right\rangle \\
& =i G^{2} m_{2}^{2} \sum_{j=0}^{\infty}\left[-2 \omega m_{1} m_{2}^{2} G_{j}^{(1,1)}\left[\left(v_{2}^{\mu}-v_{1}^{\mu} \omega\right) I_{j+2}^{\alpha}-I_{j+2}^{\mu} v_{2}^{\alpha}\right]\left\langle S_{1 \alpha}\right\rangle\right. \\
& \left.\quad-i \epsilon^{\mu}{ }_{\nu \lambda \tau} v_{1}^{\tau}\left[\delta_{\alpha}^{\nu} \delta_{\beta}^{\eta} G_{j}^{(2,1)}-\eta_{\alpha \beta} \eta^{\nu \eta} G_{j}^{(2,2)}+\eta_{\alpha \beta} \omega v_{2}^{\nu} v_{1}^{\eta} G_{j}^{(2,3)}\right] I_{j+2}^{\alpha \beta}\left\langle S_{1}^{\lambda}\right\rangle\left\langle S_{2 \eta}\right\rangle\right]+\mathcal{O}\left(\left\langle S_{1}\right\rangle^{2}\right) .
\end{aligned}
$$

We simply add eq. (5.10) and eq. (5.13) to get the full angular impulse of particle 1 in the gravitational case. Again, the map in eq. (5.6) can be applied to the gravitational impulse to obtain the electromagnetic result. The same calculation can be performed for particle 2 , but we don't give the result here as it is almost identical to the calculation for particle 1.

\section{Eikonal phase}

The eikonal phase provides an alternative means for extracting physical observables from the classical portion of scattering amplitudes, and has been successfully applied to systems involving low spins up to $\mathcal{O}\left(G^{2}\right)[8,12]$. Relations between classical observables and the eikonal phase were proposed to all perturbative orders in ref. [8]; however, as we are working with tidal effects at the leading-PM order, we only need these relations to leading order. For the linear impulse and the spin kick, they are

$$
\Delta \boldsymbol{p}_{\perp}=\nabla_{\boldsymbol{b}} \chi \quad \text { and } \quad \Delta \boldsymbol{S}_{a}^{i}=-\epsilon^{i j k} \frac{\partial \chi}{\partial \boldsymbol{S}_{a}^{j}} \boldsymbol{S}_{a}^{k}
$$

where $\chi$ is the eikonal phase, $\Delta$ indicates changes in momentum or spin, and $a=1,2$ labels the particles and is not summed over. We use Latin letters from the middle of the alphabet to denote spatial indices, which are raised and lowered with the Euclidean metric. The former relation yields the linear impulse in the plane perpendicular to the momentum at negative infinity. Choosing kinematics such that this momentum is oriented along the $z$-axis, the impulse in the parallel direction is obtained through energy conservation: $\Delta p_{z}=-(\Delta \boldsymbol{p})^{2} / 2|\boldsymbol{p}|$. Further to the results in section 5 , the eikonal phase will allow us to compute the spin kick and the scattering angle for aligned spins. First, we must compute the eikonal phase.

The eikonal phase $\chi=\chi_{1}+\chi_{2}+\ldots$ is defined as the Fourier transform of the (relativistically normalized) amplitude in the perpendicular plane described above, with the subscripts denoting contributions from the corresponding order in the coupling constant:

$$
\chi_{i}=\frac{1}{4 m_{1} m_{2} \sqrt{\omega^{2}-1}} \int \frac{d^{2-2 \epsilon} \boldsymbol{q}}{(2 \pi)^{2-2 \epsilon}} e^{-i \boldsymbol{q} \cdot \boldsymbol{b}} \mathcal{M}_{i}^{\prime}(\boldsymbol{q})
$$

where the prime on the amplitude indicates that we ignore iteration pieces. We will use $\Delta \chi$ to denote tidal contributions to the eikonal phase. The leading-PM contributions from the 
tidal deformations originate from a one-loop amplitude, so $\Delta \chi_{1}=0$ and we will calculate the $\Delta \chi_{2}$ part of the eikonal phase. This involves integrals of the type

$$
\int \frac{d^{2} \boldsymbol{q}}{(2 \pi)^{2}} e^{-i \boldsymbol{q} \cdot \boldsymbol{b}} \frac{\pi^{2}}{|\boldsymbol{q}|}\left(\frac{\boldsymbol{q}^{2}}{2}\right)^{j+2} \hat{\mathbb{O}}^{A}\left(\boldsymbol{p}, \boldsymbol{q}, \boldsymbol{S}_{a}\right)=\hat{\mathcal{O}}^{A}\left(\boldsymbol{p}, \nabla_{\boldsymbol{b}}, \boldsymbol{S}_{a}\right) I_{j+2}(\boldsymbol{b}) \frac{\mathcal{N}}{4}
$$

where we have shifted $\boldsymbol{q} \rightarrow-\boldsymbol{q}$ on the right-hand side to eliminate relative signs with the integrals in appendix B; $\mathcal{N}$ and $I_{j+2}$ are given there. The classical spin structures $\hat{\mathbb{O}}^{A}$ are listed in eq. (4.3). They have been removed from the integral by re-expressing them as differential operators in impact-parameter space acting on the integral, yielding

$$
\begin{array}{cll}
\hat{\mathcal{O}}^{(0)}=\mathbb{I}, & \hat{\mathcal{O}}^{(1,1)}=-\left(\boldsymbol{S}_{1} \times \boldsymbol{p}\right) \cdot \nabla_{\boldsymbol{b}}, & \hat{\mathcal{O}}^{(1,2)}=-\left(\boldsymbol{S}_{2} \times \boldsymbol{p}\right) \cdot \nabla_{\boldsymbol{b}}, \\
\hat{\mathcal{O}}^{(2,1)}=-\left(\boldsymbol{S}_{1} \cdot \nabla_{\boldsymbol{b}}\right)\left(\boldsymbol{S}_{2} \cdot \nabla_{\boldsymbol{b}}\right), & \hat{\mathcal{O}}^{(2,2)}=-\left(\boldsymbol{S}_{1} \cdot \boldsymbol{S}_{2}\right) \nabla_{\boldsymbol{b}}^{2}, & \hat{\mathcal{O}}^{(2,3)}=-\left(\boldsymbol{p} \cdot \boldsymbol{S}_{1}\right)\left(\boldsymbol{p} \cdot \boldsymbol{S}_{2}\right) \nabla_{\boldsymbol{b}}^{2} .
\end{array}
$$

This allows us to write the eikonal phase $\Delta \chi_{2}$ compactly as,

$$
\Delta \chi_{2}=2 G^{2} m_{2}^{2} \sum_{j=0}^{\infty} \hat{\mathcal{K}}_{j}\left(\omega, \boldsymbol{p}, \nabla_{\boldsymbol{b}}, \boldsymbol{S}_{a}\right) I_{j+2}(\boldsymbol{b}), \quad \hat{\mathcal{K}}_{j}\left(\omega, \boldsymbol{p}, \nabla_{\boldsymbol{b}}, \boldsymbol{S}_{a}\right) \equiv 4 E_{1} E_{2} \sum_{A} \Delta b_{2, j}^{A}(\omega) \hat{\mathcal{O}}^{A}
$$

where the dependence on each quantity has been written explicitly. The function $\Delta b_{2, j}^{A}(\omega)$ is either equal to $m^{A} \Delta a_{\mathrm{cov}, 2, j}^{A}(\omega)$ (no sum over $A$ ) or $\Delta a_{2, j}^{A}(\omega)$, depending on whether we work with the covariant or the canonical spin. The constant $m^{A}$ accounts for the normalization of the Dirac spinors, and is given by

$$
m^{A}= \begin{cases}4 m_{1} m_{2}, & A=(0), \\ 2 m_{2}, & A=(1,1) \\ 2 m_{1}, & A=(1,2), \\ 1, & \text { otherwise }\end{cases}
$$

We refer to the operator $\hat{\mathcal{K}}_{j}$ as the eikonal operator.

We can now substitute this form of the eikonal phase into eq. (6.1) to obtain the impulse and spin kick. For the impulse, the derivative with respect to the impact parameter commutes with the eikonal operator and only acts on the integral. By contrast, for the spin kick, the derivative with respect to the spin vector only acts on the eikonal operator: ${ }^{9}$

$$
\begin{aligned}
& \Delta \boldsymbol{p}_{\perp}=-2 G^{2} m_{2}^{2} \sum_{j=0}^{\infty} \hat{\mathcal{K}}_{j}\left(\omega, \boldsymbol{p}, \nabla_{\boldsymbol{b}}, \boldsymbol{S}_{a}\right)\left(\nabla_{\boldsymbol{b}} I_{j+2}(\boldsymbol{b})\right), \\
& \Delta \boldsymbol{S}_{a}=-2 G^{2} m_{2}^{2} \sum_{j=0}^{\infty}\left(\frac{\partial \hat{\mathcal{K}}_{j}\left(\omega, \boldsymbol{p}, \nabla_{\boldsymbol{b}}, \boldsymbol{S}_{a}\right)}{\partial \boldsymbol{S}_{a}} \times \boldsymbol{S}_{a}\right) I_{j+2}(\boldsymbol{b}) .
\end{aligned}
$$

\footnotetext{
${ }^{9}$ The sign for the linear impulse here is because we compute the impulse for the emitting particle.
} 
Setting $\Delta b_{2, j}^{A}(\omega)=m^{A} \Delta a_{\mathrm{cov}, 2, j}^{A}(\omega)$, the linear impulse calculated from the eikonal phase agrees with eq. (5.4). We find the spin kick for particle 1 to be

$$
\begin{aligned}
\Delta \boldsymbol{S}_{1}^{k}= & -8 E_{1} E_{2} G^{2} m_{2}^{2} \sum_{j=0}^{\infty}\left\{-i \Delta b_{2, j}^{(1,1)}\left[-\boldsymbol{p}^{k} \boldsymbol{S}_{1}^{m}+\left(\boldsymbol{p} \cdot \boldsymbol{S}_{1}\right) \delta^{k m}\right] I_{j+2}^{m}\right. \\
& \left.+\left[-\Delta b_{2, j}^{(2,1)}\left(\varepsilon^{k \ell m} \boldsymbol{S}_{1}^{\ell}\right) \boldsymbol{S}_{2}^{n}-\Delta b_{2, j}^{(2,2)}\left(\boldsymbol{S}_{1} \times \boldsymbol{S}_{2}\right)^{k} \delta^{m n}+\Delta b_{2, j}^{(2,3)}\left(\boldsymbol{p} \times \boldsymbol{S}_{1}\right)^{k}\left(\boldsymbol{p} \cdot \boldsymbol{S}_{2}\right) \delta^{m n}\right] I_{j+2}^{m n}\right\} .
\end{aligned}
$$

This expression for the spin kick is valid in the center-of-mass frame. Computing the same quantity in the rest frame of particle 1, we find agreement with the commutator portion of the angular impulse, eq. (5.13).

From the eikonal phase it is also possible to obtain the scattering angle. In the case of two non-rotating bodies, the dynamics are constrained to a plane, so a unique scattering angle can be defined. Spinning particles, however, introduce precession effects, which are described by an additional angle. This additional complication can be ignored in the special case of aligned spins, which again restricts motion to a plane. We will calculate the unique scattering angle in this special case, where the spin structures are

$$
\boldsymbol{S}_{1} \cdot \boldsymbol{S}_{2}=\left|\boldsymbol{S}_{1}\right|\left|\boldsymbol{S}_{2}\right|, \quad \boldsymbol{b} \cdot \boldsymbol{S}_{i}=0, \quad \boldsymbol{p} \cdot \boldsymbol{S}_{i}=0 .
$$

This scattering angle can be related to the eikonal phase using the stationary phase approximation [66]:

$$
2 \sin \frac{\theta}{2} \approx \theta=-\frac{1}{|\boldsymbol{p}|} \frac{\partial}{\partial|\boldsymbol{b}|} \chi(\omega, \boldsymbol{b}) .
$$

For the calculation of the scattering angle, we will use the eikonal phase in terms of the canonical spin, as it is to be compared to the scattering angle derived from canonical equations of motion. Thus, $\Delta b_{2, j}^{A}(\omega)=\Delta a_{2, j}^{A}(\omega)$.

As in the case of the linear impulse, the derivative with respect to $|\boldsymbol{b}|$ acts only on the integral. Using the relation

$$
\frac{\partial}{\partial|\boldsymbol{b}|} I_{j+2}(\boldsymbol{b})=\frac{2|\boldsymbol{b}|}{(5+2 j)} I_{j+3}(\boldsymbol{b}),
$$

we can write the scattering angle generally as

$$
\Delta \theta_{2}=-\frac{2 G^{2} m_{2}^{2}}{|\boldsymbol{p}|} \sum_{j=0}^{\infty} \frac{2|\boldsymbol{b}|}{(5+2 j)} \hat{\mathcal{K}}_{j}\left(\omega, \boldsymbol{p}, \nabla_{\boldsymbol{b}}, \boldsymbol{S}_{a}\right) I_{j+3}(\boldsymbol{b}) .
$$

Using the action of each differential operator on the integral (eq. (B.23)), the tidal corrections to the $2 \mathrm{PM}$ aligned-spin scattering angle are

$$
\begin{aligned}
\Delta \theta_{2}= & \frac{\pi G^{2} m_{2} E_{1} E_{2}}{|\boldsymbol{p}| m_{1} \sqrt{\omega^{2}-1}} \sum_{j=0}^{\infty} \frac{\Gamma[9 / 2+j]}{\Gamma[-3 / 2-j]}\left(\frac{2}{\boldsymbol{b}^{2}}\right)^{j+4} \\
& \times\left[\Delta a_{2, j}^{(0)} \frac{\boldsymbol{b}^{2}}{(7+2 j)}+\Delta a_{2, j}^{(1,1)}\left(\boldsymbol{S}_{1} \times \boldsymbol{p}\right) \cdot \boldsymbol{b}+\Delta a_{2, j}^{(1,2)}\left(\boldsymbol{S}_{2} \times \boldsymbol{p}\right) \cdot \boldsymbol{b}\right. \\
& \left.+\left(\Delta a_{2, j}^{(2,1)}-(5+2 j) \Delta a_{2, j}^{(2,2)}\right)\left|\boldsymbol{S}_{1}\right|\left|\boldsymbol{S}_{2}\right|\right] .
\end{aligned}
$$


The spin-monopole portion of this is in agreement with that in ref. [35], upon applying the matching conditions in eqs. (3.23) and (3.24).

\section{Conclusion}

As the recent burst in activity suggests, quantum-field-theoretic techniques are well suited for studying tidal deformations, where the tidal effects are characterized by higherdimensional operators. A full classification of tidal operators relevant for tidally-deformed spinless objects at the one-loop level was presented in ref. [35], and in this paper we have extended this analysis to include effects at linear order in the spin of the deformed object. As in ref. [35], the starting point was the Hilbert series, which counts the number of independent operators - equivalently, the number of independent amplitudes - for a given field content and number of covariant derivatives. Using this as a guide, we wrote down both the amplitude basis and the operator basis for a spin- $1 / 2$ particle coupled to photons or gravitons through at most two photon field strengths/Weyl tensors. These operator bases represent the full set of operators coupling two spinors to two photon field strengths or Weyl tensors, describing the complete set of finite-size contributions at one loop.

Employing traditional Feynman diagrammatic methods, we used these actions to calculate the one-loop amplitudes - corresponding to the leading-PM order in the case of gravity - for these finite-size effects. We find that the spin-multipole universality for long-range classical effects observed in refs. [62, 63] extends to tidally deformed systems; the spin-monopole portions of the amplitudes calculated here are in agreement with those found in ref. [35]. For general Wilson coefficients, the finite-size contributions to the amplitudes break the observed correspondence between the $q \cdot S_{1} q \cdot S_{2}$ and $q^{2} S_{1} \cdot S_{2}$ terms in the point-particle case.

We then extracted various classical quantities from these amplitudes. First, we extended the EFT matching formalism of ref. [8] to include tidal effects, and subsequently used this formalism to derive the tidal corrections to the conservative gravitational Hamiltonian at leading-PM order. We then derived the finite-size contributions to the electromagnetic and gravitational linear and angular impulse. The linear impulse was computed in two ways for the gravitational case, producing the same result: it was first calculated using the formalism of ref. [4], then through application of the eikonal phase [8]. The angular impulse was computed using the method of ref. [6]. A portion of this result was corroborated by the extraction of the spin kick from the eikonal phase; when computing this quantity in the rest frame of particle 1, it agrees with the commutator contribution to the angular impulse. Finally, the eikonal phase allowed us to derive the scattering angle in the case of aligned spins.

In the interest of describing real macroscopic systems, one must account for finite-size effects at arbitrary orders in the spin vector. We have demonstrated that EFT techniques such as the Hilbert series and the construction of on-shell helicity amplitudes are suitable for combining spin and finite-size effects. These techniques can yet be extended to higher spins, but this is outside the scope of this paper. 
The description of finte-size effects for spinning particles also opens the door for the study of the entanglement entropy generated in the scattering of tidally deformed objects. A similar analysis to that performed in ref. [67] can be applied to the systems described here, potentially shedding some light on the values of tidal Love numbers for Kerr black holes in a general (but weak) gravitational environment.

Finally, it would be helpful to understand links between the tidal action here and worldline actions describing tidal effects at linear order in the angular momentum, perhaps by taking the heavy or non-relativistic limits of the quantum action in eq. (2.14). The action in eq. (2.14) is the most general, non-redundant action describing parity-even fourpoint contact terms, but not all operators necessarily need to contribute classical effects; for example, we found that certain operators enter only at subleading orders at the oneloop level. A matching to classical quantities is then essential for determining the subset of operators that do indeed contribute classically.

\section{Acknowledgments}

We thank Clifford Cheung, Andrés Luna, Ben Maybee, and Julio Parra-Martinez for related discussions. This project has received funding from the European Union's Horizon 2020 research and innovation programme under the Marie Skłodowska-Curie grant agreement No. 764850 "SAGEX" and by the F.R.S.-FNRS with the EOS — be.h project n. 30820817. A.H. is supported by the DOE under grant no. DE-SC0011632 and by the Walter Burke Institute for Theoretical Physics.

\section{A Loop integrals}

For the calculation of tidal effects at one loop, we only need to evaluate the triangle integral, but we need to do so for arbitrary even rank,

$$
\mathcal{I}_{\triangleleft}^{\mu_{1} \ldots \mu_{2 k}} \equiv \int \frac{d^{4} l}{(2 \pi)^{4}} \frac{l^{\mu_{1} \ldots \mu_{2 k}}}{l^{2}(l+q)^{2}\left[-v_{2} \cdot l+i \epsilon\right]} .
$$

The Passarino-Veltman reduction [68] allows one to solve this for any rank in terms of the scalar triangle integral ${ }^{10}$

$$
\mathcal{I}_{\triangleleft} \equiv \int \frac{d^{4} l}{(2 \pi)^{4}} \frac{1}{l^{2}(l+q)^{2}\left[-v_{2} \cdot l+i \epsilon\right]}=-\frac{i S}{16 \pi^{2}},
$$

where $S \equiv \pi^{2} / \sqrt{-q^{2}}$, but this becomes cumbersome for high ranks.

Fortunately, the portions of the integrals needed for our purposes are only the leadingin- $\hbar$ pieces. When the tensor integral is dotted into $v_{1 \mu_{1} \ldots \mu_{2 k}}$, ref. [35] found by explicit calculation up to rank $2 k=10$ that this leading term is

$$
v_{1 \mu_{1} \ldots \mu_{2 k}} \mathcal{I}_{\triangleleft}^{\mu_{1} \ldots \mu_{2 k}} \equiv \int \frac{d^{4} l}{(2 \pi)^{4}} \frac{\left(v_{1} \cdot l\right)^{2 k}}{l^{2}(l+q)^{2}\left[-v_{2} \cdot l+i \epsilon\right]}=\frac{\left(\frac{1}{2}\right)_{k}}{4^{k}(1)_{k}}\left(\omega^{2}-1\right)^{k} q^{2 k} \mathcal{I}_{\triangleleft}+\mathcal{O}\left(q^{2 k}\right) .
$$

\footnotetext{
${ }^{10}$ More precisely, the Passarino-Veltman reduction expresses the rank- $2 k$ integral in terms of scalar triangle, bubble, and tadpole integrals, but neither of the latter two contributes classical information.
} 
This formula was proven for general $k$ in ref. [37] by finding the residue of the matter pole and then calculating the remaining three-dimensional integral using a known expression [69].

When calculating the leading-PM amplitudes for spinning tidal effects, we also need five additional integrals:

$$
\begin{aligned}
& \left(\bar{u}_{2} q^{\alpha} \sigma_{\alpha \mu_{1}} u_{1}\right) v_{1 \mu_{2} \ldots \mu_{2 k}} \mathcal{I}_{\triangleleft}^{\mu_{1} \ldots \mu_{2 k}}, \\
& \left(\bar{u}_{4} q^{\alpha} \sigma_{\alpha \mu_{1}} u_{3}\right) v_{1 \mu_{2} \ldots \mu_{2 k}} \mathcal{I}_{\triangleleft}^{\mu_{1} \ldots \mu_{2 k}}, \\
& \left(\bar{u}_{2} q^{\alpha} \sigma_{\alpha \mu_{1}} u_{1}\right)\left(\bar{u}_{4} q^{\beta} \sigma_{\beta \mu_{2}} u_{3}\right) v_{1 \mu_{3} \ldots \mu_{2 k}} \mathcal{I}_{\triangleleft}^{\mu_{1} \ldots \mu_{2 k}}, \\
& S_{1 \mu_{1}} S_{2 \mu_{2}} v_{1 \mu_{3} \ldots \mu_{2 k}} \mathcal{I}_{\triangleleft}^{\mu_{1} \ldots \mu_{2 k}}, \\
& q \cdot S_{1} S_{2 \mu_{1}} v_{1 \mu_{2} \ldots \mu_{2 k+1}} \mathcal{I}_{\triangleleft}^{\mu_{1} \ldots \mu_{2 k+1}}=S_{1 \mu_{1}} q \cdot S_{2} v_{1 \mu_{2} \ldots \mu_{2 k+1}} \mathcal{I}_{\triangleleft}^{\mu_{1} \ldots \mu_{2 k+1}} .
\end{aligned}
$$

The leading terms for these integrals are

$$
\begin{aligned}
& \left(\bar{u}_{2} q^{\alpha} \sigma_{\alpha \mu_{1}} u_{1}\right) v_{1 \mu_{2} \ldots \mu_{2 k}} \mathcal{I}_{\triangleleft}^{\mu_{1} \ldots \mu_{2 k}}=\left(\bar{u}_{2} q^{\alpha} v_{2}^{\beta} \sigma_{\alpha \beta} u_{1}\right) \frac{\left(\frac{1}{2}\right)_{k}}{4^{k}(1)_{k}} \omega\left(\omega^{2}-1\right)^{k-1} q^{2 k} \mathcal{I}_{\triangleleft}, \\
& \left(\bar{u}_{4} q^{\alpha} \sigma_{\alpha \mu_{1}} u_{3}\right) v_{1 \mu_{2} \ldots \mu_{2 k}} \mathcal{I}_{\triangleleft}^{\mu_{1} \ldots \mu_{2 k}}=-\left(\bar{u}_{4} q^{\alpha} v_{1}^{\beta} \sigma_{\alpha \beta} u_{3}\right) \frac{\left(\frac{1}{2}\right)_{k}}{4^{k}(1)_{k}}\left(\omega^{2}-1\right)^{k-1} q^{2 k} \mathcal{I}_{\triangleleft}, \\
& \left(\bar{u}_{2} q^{\alpha} \sigma_{\alpha \mu_{1}} u_{1}\right)\left(\bar{u}_{4} q^{\beta} \sigma_{\beta \mu_{2}} u_{3}\right) v_{1 \mu_{3} \ldots \mu_{2 k}} \mathcal{I}_{\triangleleft}^{\mu_{1} \ldots \mu_{2 k}}= \\
& \left(\bar{u}_{2} q^{\alpha} \sigma_{\alpha \mu} u_{1}\right)\left(\bar{u}_{4} q^{\beta} \sigma_{\beta \nu} u_{3}\right) \frac{\left(\frac{1}{2}\right)_{k}}{4^{k}(1)_{k}} q^{2 k} \mathcal{I}_{\triangleleft} \\
& \times\left[-\frac{1}{2 k-1} \eta^{\mu \nu}\left(\omega^{2}-1\right)^{k-1}-\frac{2(k-1)}{(2 k-1)} v_{1}^{\nu} v_{2}^{\mu} \omega\left(\omega^{2}-1\right)^{k-2}\right], \\
& S_{1 \mu_{1}} S_{2 \mu_{2}} v_{1 \mu_{3} \ldots \mu_{2 k}} \mathcal{I}_{\triangleleft}^{\mu_{1} \ldots \mu_{2 k}}=S_{1 \mu_{1}} S_{2 \nu} \frac{\left(\frac{1}{2}\right)_{k}}{4^{k}(1)_{k}} q^{2 k} \mathcal{I}_{\triangleleft} \\
& \times\left[\frac{2 k+1}{2 k-1} q^{\mu} q^{\nu}\left(\omega^{2}-1\right)^{k-1}-\frac{1}{2 k-1} q^{2} \eta^{\mu \nu}\left(\omega^{2}-1\right)^{k-1}\right. \\
& \left.-\frac{2(k-1)}{(2 k-1)} q^{2} v_{1}^{\nu} v_{2}^{\mu} \omega\left(\omega^{2}-1\right)^{k-2}\right], \\
& q \cdot S_{1} S_{2 \mu_{1}} v_{1 \mu_{2} \ldots \mu_{2 k-1}} \mathcal{I}_{\triangleleft}^{\mu_{1} \ldots \mu_{2 k-1}}=-q \cdot S_{1} q \cdot S_{2} \frac{\left(\frac{1}{2}\right)_{k-1}}{2^{2 k-1}(1)_{k-1}}\left(\omega^{2}-1\right)^{k-1} q^{2 k-2} \mathcal{I}_{\triangleleft} .
\end{aligned}
$$

where $k \geq 1$. The first three of these contractions rely on the same portion of the rank- $2 k$ triangle integral as that of eq. (A.3); namely, only the part of the integral whose tensor structure contains no transfer momenta. As such, these integrals can be derived by a combinatoric analysis of eq. (A.3). We show now this combinatoric analysis.

The leading-in- $\hbar$ portion of the triangle integral proportional to tensor structures containing only factors of the metric and the velocity can be inferred from eq. (A.3). To do this, we expand the binomial $\left(\omega^{2}-1\right)^{k}$ and "uncontract" the integral by noting that 


$$
\begin{aligned}
\omega & =v_{1 \mu} v_{2}^{\mu} \text { and } v_{1 \mu \nu} \eta^{\mu \nu}=1: \\
& v_{1 \mu_{1} \ldots \mu_{2 k}} \mathcal{I}_{\triangleleft}^{\mu_{1} \ldots \mu_{2 k}} \\
& =\frac{\left(\frac{1}{2}\right)_{k}}{4^{k}(1)_{k}} q^{2 k} \mathcal{I}_{\triangleleft} \sum_{n=0}^{k}\left(\begin{array}{l}
k \\
n
\end{array}\right)(-1)^{k-n} v_{1 \mu_{1} \ldots \mu_{2 k-2 n}} \eta^{\mu_{1} \mu_{2}, \ldots, \mu_{2 k-2 n-1} \mu_{2 k-2 n}} v_{1 \alpha_{1} \ldots \alpha_{2 n}} v_{2}^{\alpha_{1} \ldots \alpha_{2 n}} \\
& =v_{1 \mu_{1} \ldots \mu_{2 k}}\left[\frac{\left(\frac{1}{2}\right)_{k}}{4^{k}(1)_{k}} q^{2 k} \mathcal{I}_{\triangleleft} \sum_{n=0}^{k} \frac{1}{(2 k) !}\left(\begin{array}{l}
k \\
n
\end{array}\right)(-1)^{k-n} \eta^{\left\{\mu_{1} \mu_{2}, \ldots, \mu_{2 k-2 n-1} \mu_{2 k-2 n}\right.} v_{2}^{\left.\mu_{2 k-2 n+1} \ldots \mu_{2 k}\right\}}\right],
\end{aligned}
$$

where $\eta^{\mu_{1} \mu_{2}, \ldots, \mu_{2 k-1} \mu_{2 k}} \equiv \eta^{\mu_{1} \mu_{2}} \ldots \eta^{\mu_{2 k-1} \mu_{2 k}}$. We now identify the quantity in the square brackets with the uncontracted triangle integral. Curly brackets denote symmetrization without normalization. Note that this is not the actual value of the uncontracted triangle integral: even at the order of $\hbar$ at which we are working, tensor structures with an even number of transfer-momentum four-vectors are present. For our purposes, however, these contributions will always vanish or become subleading through the on-shell condition $v_{1} \cdot q \sim$ $q^{2} / m$ when contracted with the spin structures in eqs. (A.4) to (A.6).

We begin with the contraction in eq. (A.4). Eq. (3.12) implies that the $n=0$ term vanishes, and that the only non-vanishing terms have a $v_{2}^{\mu}$ contracted with the sigma matrix. The remaining indices will all be contracted symmetrically with factors of $v_{1 \mu}$, so for a term with $2 n$ factors of $v_{2}^{\mu}$ and $2 k$ total symmetrized Lorentz indices there will be $2 n(2 k-1)$ ! non-vanishing and identical distributions of the Lorentz indices. Then,

$$
\begin{aligned}
& \left(\bar{u}_{2} q^{\alpha} \sigma_{\alpha \mu_{1}} u_{1}\right) v_{1 \mu_{2} \ldots \mu_{2 k}} \mathcal{I}_{\triangleleft}^{\mu_{1} \ldots \mu_{2 k}} \\
& \quad=\left(\bar{u}_{2} q^{\alpha} v_{2}^{\mu_{2 k}} \sigma_{\alpha \mu_{2 k}} u_{2 k}\right) \frac{\left(\frac{1}{2}\right)_{k}}{4^{k}(1)_{k}} q^{2 k} \mathcal{I}_{\triangleleft} \sum_{n=1}^{k} \frac{2 n(2 k-1) !}{(2 k) !}\left(\begin{array}{c}
k \\
n
\end{array}\right)(-1)^{k-n} \omega^{2 n-1} \\
& \quad=\left(\bar{u}_{2} q^{\alpha} v_{2}^{\mu_{2 k}} \sigma_{\alpha \mu_{2 k}} u_{2 k}\right) \frac{\left(\frac{1}{2}\right)_{k}}{4^{k}(1)_{k}} q^{2 k} \mathcal{I}_{\triangleleft} \omega \sum_{n=0}^{k-1}\left(\begin{array}{c}
k-1 \\
n
\end{array}\right)(-1)^{k-1-n} \omega^{2 n-2} \\
& \quad=\left(\bar{u}_{2} q^{\alpha} v_{2}^{\mu_{2 k}} \sigma_{\alpha \mu_{2 k}} u_{2 k}\right) \frac{\left(\frac{1}{2}\right)_{k}}{4^{k}(1)_{k}} \omega\left(\omega^{2}-1\right)^{k-1} q^{2 k} \mathcal{I}_{\triangleleft} .
\end{aligned}
$$

This is what we wanted to prove.

The argument for the contraction in eq. (A.5) is similar. However, because of eq. (3.13), now only terms with at least one metric survive. Thus the $n=k$ term vanishes, and a term with $k-n$ metrics and $2 k$ total symmetrized Lorentz indices contributes $2(k-n)(2 k-1)$ ! identical Lorentz index distributions. Therefore,

$$
\begin{aligned}
& \left(\bar{u}_{4} q^{\alpha} v_{1}^{\mu_{1}} \sigma_{\alpha \mu_{1}} u_{3}\right) v_{1 \mu_{2} \ldots \mu_{2 k}} \mathcal{I}_{\triangleleft}^{\mu_{1} \ldots \mu_{2 k}} \\
& =\left(\bar{u}_{4} q^{\alpha} v_{1}^{\mu_{2 k}} \sigma_{\alpha \mu_{2 k}} u_{3}\right) \frac{\left(\frac{1}{2}\right)_{k}}{4^{k}(1)_{k}} q^{2 k} \mathcal{I}_{\triangleleft} \sum_{n=0}^{k-1} \frac{2(k-n)(2 k-1) !}{(2 k) !}\left(\begin{array}{c}
k \\
k-n
\end{array}\right)(-1)^{k-n} \omega^{2 n} .
\end{aligned}
$$


We have used the identity $\left(\begin{array}{l}k \\ n\end{array}\right)=\left(\begin{array}{c}k \\ k-n\end{array}\right)$. Inverting the order of the sum,

$$
\begin{aligned}
& \left(\bar{u}_{4} q^{\alpha} v_{1}^{\mu_{1}} \sigma_{\alpha \mu_{1}} u_{3}\right) v_{1 \mu_{2} \ldots \mu_{2 k}} \mathcal{I}_{\triangleleft}^{\mu_{1} \ldots \mu_{2 k}} \\
& \quad=\left(\bar{u}_{4} q^{\alpha} v_{1}^{\mu_{2 k}} \sigma_{\alpha \mu_{2 k}} u_{3}\right) \frac{\left(\frac{1}{2}\right)_{k}}{4^{k}(1)_{k}} q^{2 k} \mathcal{I}_{\triangleleft} \sum_{n=1}^{k} \frac{2 n(2 k-1) !}{(2 k) !}\left(\begin{array}{c}
k \\
n
\end{array}\right)(-1)^{n}\left(\omega^{2}\right)^{k-n} \\
& \quad=\left(\bar{u}_{4} q^{\alpha} v_{1}^{\mu_{2 k}} \sigma_{\alpha \mu_{2 k}} u_{3}\right) \frac{\left(\frac{1}{2}\right)_{k}}{4^{k}(1)_{k}} q^{2 k} \mathcal{I}_{\triangleleft} \sum_{n=0}^{k-1}\left(\begin{array}{c}
k-1 \\
n
\end{array}\right)(-1)^{n+1}\left(\omega^{2}\right)^{k-1-n} \\
& \quad=-\left(\bar{u}_{4} q^{\alpha} v_{1}^{\mu_{2 k}} \sigma_{\alpha \mu_{2 k}} u_{3}\right) \frac{\left(\frac{1}{2}\right)_{k}}{4^{k}(1)_{k}}\left(\omega^{2}-1\right)^{k-1} q^{2 k} \mathcal{I}_{\triangleleft},
\end{aligned}
$$

as claimed.

Finally, we move to the contraction of eq. (A.6). Eq. (3.13) implies that the $n=k$ term in the sum vanishes, and that we must always have one metric contracted with the spin of particle 2. There are then clearly two types of contributions: those where the second index of that metric contracts with the spin of particle 1 , and those where it contracts with a factor of $v_{1}^{\mu}$ :

$$
\begin{aligned}
& \left(\bar{u}_{2} q^{\alpha} \sigma_{\alpha \mu_{1}} u_{1}\right)\left(\bar{u}_{4} q^{\beta} \sigma_{\beta \mu_{2}} u_{3}\right) v_{1 \mu_{3} \ldots \mu_{2 k}} \mathcal{I}_{\triangleleft}^{\mu_{1} \ldots \mu_{2 k}} \\
& =\left(\bar{u}_{2} q^{\alpha} \sigma_{\alpha \mu_{1}} u_{1}\right)\left(\bar{u}_{4} q^{\beta} \sigma_{\beta \mu_{2}} u_{3}\right) v_{1 \mu_{3} \ldots \mu_{2 k}} \frac{\left(\frac{1}{2}\right)_{k}}{4^{k}(1)_{k}} q^{2 k} \mathcal{I}_{\triangleleft} \\
& \times\left[\eta^{\mu_{1} \mu_{2}} \sum_{n=0}^{k-1} \frac{N_{n}}{(2 k) !}\left(\begin{array}{l}
k \\
n
\end{array}\right)(-1)^{k-n} \eta^{\left\{\mu_{3} \mu_{4}, \ldots, \mu_{2 k-2 n-1} \mu_{2 k-2 n}\right.} v_{2}^{\left.\mu_{2 k-2 n+1} \ldots \mu_{2 k}\right\}}\right. \\
& \left.\quad+\eta^{\mu_{2} \mu_{3}} \sum_{n=0}^{k-1} \frac{P_{n}}{(2 k) !}\left(\begin{array}{l}
k \\
n
\end{array}\right)(-1)^{k-n} \eta^{\left\{\mu_{1} \mu_{4}, \ldots, \mu_{2 k-2 n-1} \mu_{2 k-2 n}\right.} v_{2}^{\left.\mu_{2 k-2 n+1} \ldots \mu_{2 k}\right\}}\right] .
\end{aligned}
$$

Our task is now to determine the integers $N_{n}$ and $P_{n}$ - which count the number of identical Lorentz index distributions that do not vanish when contracted with the spin structure and to resum the sums. In the case of $N_{n}$, in a term with $k-n$ metrics, there are $k-n$ metrics that can be contracted with the spin of particle 2 , two identical ways to distribute the indices on this metric, and $(2 k-2)$ ! identical ways to distribute the remaining Lorentz indices for each of these metric permutations. Therefore, $N_{n}=2(k-n)(2 k-2)$ !.

Now, in the term corresponding to $P_{n}$, eq. (3.12) implies that the $n=0$ term also vanishes, and that one factor of $v_{2}^{\mu}$ must be contracted with the spin of particle 1 . For a term with $2 n$ factors of $v_{2}^{\mu}$, there are then $2 n$ identical ways to contract a velocity with the spin. Moreover, such a term will have $k-n$ metrics that can be contracted with the spin of particle 2, each of which has two indices that can be contracted in this way. Finally, as eqs. (3.12) and (3.13) fix two indices, there are $(2 k-2)$ ! identical permutations of the remaining Lorentz indices. Therefore, $P_{n}=(2 n)[2(k-n)](2 k-2)$ !. 
Putting these together,

$$
\begin{aligned}
& \left(\bar{u}_{2} q^{\alpha} \sigma_{\alpha \mu_{1}} u_{1}\right)\left(\bar{u}_{4} q^{\beta} \sigma_{\beta \mu_{2}} u_{3}\right) v_{1 \mu_{3} \ldots \mu_{2 k}} \mathcal{I}_{\triangleleft}^{\mu_{1} \ldots \mu_{2 k}} \\
& =\left(\bar{u}_{2} q^{\alpha} \sigma_{\alpha \mu_{1}} u_{1}\right)\left(\bar{u}_{4} q^{\beta} \sigma_{\beta \mu_{2}} u_{3}\right) \frac{\left(\frac{1}{2}\right)_{k}}{4^{k}(1)_{k}} q^{2 k} \mathcal{I}_{\triangleleft} \\
& \quad \times\left[\eta^{\mu_{1} \mu_{2}} \sum_{n=0}^{k-1} \frac{(k-n)}{k(2 k-1)}\left(\begin{array}{c}
k \\
n
\end{array}\right)(-1)^{k-n}\left(\omega^{2}\right)^{n}\right. \\
& \left.\quad+\eta^{\mu_{2} \mu_{3}} v_{1 \mu_{3}} v_{2}^{\mu_{1}} \omega \sum_{n=0}^{k-2} \frac{2(n+1)(k-1-n)}{k(2 k-1)}\left(\begin{array}{c}
k \\
n+1
\end{array}\right)(-1)^{k-1-n}\left(\omega^{2}\right)^{n}\right] .
\end{aligned}
$$

We recognize the first sum as the one in eq. (A.16) divided by $2 k-1$. Focusing on the second sum, we apply the recursive identity for the binomial coefficients, invert the sum, multiply by $(k-1) /(k-1)$, and apply the recursive identity again to find

$$
\begin{aligned}
& \left(\bar{u}_{2} q^{\alpha} \sigma_{\alpha \mu_{1}} u_{1}\right)\left(\bar{u}_{4} q^{\beta} \sigma_{\beta \mu_{2}} u_{3}\right) v_{1 \mu_{3} \ldots \mu_{2 k}} \mathcal{I}_{\triangleleft}^{\mu_{1} \ldots \mu_{2 k}} \\
& =\left(\bar{u}_{2} q^{\alpha} \sigma_{\alpha \mu} u_{1}\right)\left(\bar{u}_{4} q^{\beta} \sigma_{\beta \nu} u_{3}\right) \frac{\left(\frac{1}{2}\right)_{k}}{4^{k}(1)_{k}} q^{2 k} \mathcal{I}_{\triangleleft} \\
& \quad \times\left[-\frac{\eta^{\mu \nu}}{(2 k-1)}\left(\omega^{2}-1\right)^{k-1}+v_{1}^{\nu} v_{2}^{\mu} \omega \sum_{n=0}^{k-2} \frac{2(k-1)}{(2 k-1)}\left(\begin{array}{c}
k-2 \\
n
\end{array}\right)(-1)^{n+1}\left(\omega^{2}\right)^{k-n-2}\right] \\
& =\left(\bar{u}_{2} q^{\alpha} \sigma_{\alpha \mu} u_{1}\right)\left(\bar{u}_{4} q^{\beta} \sigma_{\beta \nu} u_{3}\right) \frac{\left(\frac{1}{2}\right)_{k}}{4^{k}(1)_{k}} q^{2 k} \mathcal{I}_{\triangleleft} \\
& \quad \times\left[-\frac{\eta^{\mu \nu}}{(2 k-1)}\left(\omega^{2}-1\right)^{k-1}-\frac{2(k-1)}{(2 k-1)} v_{1}^{\nu} v_{2}^{\mu} \omega\left(\omega^{2}-1\right)^{k-2}\right] .
\end{aligned}
$$

This completes the proof.

All integrals in this section were checked explicitly up to rank $2 k=10$.

\section{B Integrals for classical impulses and the potential}

We give here details about the integrals needed to Fourier transform the potential in section 4 to position space, as well as the integrals used to derive the linear and angular impulses in sections 5 and 6.

\section{B.1 Fourier transforms}

To convert the momentum space tidal potential in section 4 to position space, we need knowledge of three-dimensional Fourier integrals up to rank 2:

$$
J_{j}^{i_{1} \ldots i_{k}}=-\frac{1}{\sqrt{2}} \int \hat{d}^{3} \boldsymbol{q} e^{-i \boldsymbol{q} \cdot \boldsymbol{r}}\left(\frac{\boldsymbol{q}^{2}}{2}\right)^{j+3 / 2} \boldsymbol{q}^{i_{1} \ldots i_{k}}, \quad k \leq 2 .
$$

First, for $k=0$,

$$
J_{j}=2\left(\frac{2}{r^{2}}\right)^{j+3} \frac{\Gamma(j+3)}{(4 \pi)^{3 / 2} \Gamma(-j-3 / 2)},
$$


where $r \equiv|\boldsymbol{r}|$. In the rank 1 case,

$$
J_{j}^{i}=i \frac{\partial}{\partial \boldsymbol{r}^{i}} J_{j}=-2 i \boldsymbol{r}^{i}\left(\frac{2}{r^{2}}\right)^{j+4} \frac{\Gamma(j+4)}{(4 \pi)^{3 / 2} \Gamma(-j-3 / 2)} .
$$

Similarly, for rank 2 ,

$$
J_{j}^{i k}=i \frac{\partial}{\partial \boldsymbol{r}^{k}} J_{j}^{i}=2\left(\frac{2}{r^{2}}\right)^{j+4}\left[\delta^{i k}-2(j+4) \frac{\boldsymbol{r}^{i} \boldsymbol{r}^{k}}{r^{2}}\right] \frac{\Gamma(j+4)}{(4 \pi)^{3 / 2} \Gamma(-j-3 / 2)} .
$$

\section{B.2 Linear and angular impulse}

The calculation of the classical impulses in sections 5 and 6 needed the evaluation of integrals of the form

$$
I_{j+2}^{\mu_{1} \ldots \mu_{k}}(b)=\int \hat{d}^{4} q \hat{\delta}\left(2 p_{1} \cdot q\right) \hat{\delta}\left(2 p_{2} \cdot q\right) e^{-i b \cdot q} q^{\mu_{1} \ldots \mu_{k}} S\left(-\frac{q^{2}}{2}\right)^{j+2}
$$

where $\hat{d}^{n} q=d^{n} q /(2 \pi)^{n}$ and $\hat{\delta}(x)=2 \pi \delta(x)$. These integrals are similar to integrals evaluated in refs. [4, 6], and we will use the same steps here. We go to the rest frame of particle 1 , where $v_{1}=(1,0,0,0)$ and $v_{2}=(\omega, 0,0, \omega \beta)$, with $\beta$ satisfying $\omega^{2}\left(1-\beta^{2}\right)=1$. After evaluating the delta functions, we find that

$$
I_{j+2}^{\mu_{1} \ldots \mu_{k}}(\boldsymbol{b})=\frac{1}{\mathcal{N}} \int d^{2} q e^{i \boldsymbol{b} \cdot \boldsymbol{q}} q^{\mu_{1} \ldots \mu_{k}} \frac{1}{|\boldsymbol{q}|}\left(\frac{\boldsymbol{q}^{2}}{2}\right)^{j+2}, \quad \text { where } \quad \mathcal{N} \equiv 16 m_{1} m_{2} \sqrt{\omega^{2}-1}
$$

We will omit the argument of the integral from now on. We parametrize $\boldsymbol{q}$ to be in the 2-dimensional plane perpendicular to $v_{1}$ and $v_{2}, q^{\mu}=(0, \chi \cos \theta, \chi \sin \theta, 0)$, such that the rank-0 integral becomes

$$
\begin{aligned}
I_{j+2} & =\frac{1}{\mathcal{N}} \int d^{2} q e^{i \boldsymbol{b} \cdot \boldsymbol{q}} \frac{1}{|\boldsymbol{q}|}\left(\frac{\boldsymbol{q}^{2}}{2}\right)^{j+2} \\
& =\frac{1}{\mathcal{N}} \int_{0}^{\infty} d \chi \chi \int_{-\pi}^{\pi} d \theta e^{i|\boldsymbol{b}| \chi \cos \theta} \frac{1}{\chi}\left(\frac{\chi^{2}}{2}\right)^{j+2} \\
& =\frac{2 \pi}{\mathcal{N}} \int_{0}^{\infty} d \chi J_{0}(|\boldsymbol{b}| \chi)\left(\frac{\chi^{2}}{2}\right)^{j+2} \\
& =\frac{\pi}{8 m_{1} m_{2} \sqrt{\omega^{2}-1}} \frac{1}{|\boldsymbol{b}|}\left(-\frac{2}{b^{2}}\right)^{j+2} \frac{\Gamma[5 / 2+j]}{\Gamma[-3 / 2-j]}
\end{aligned}
$$

where in the last step we restored Lorentz invariance. The rank-1 integral is also straightforward to evaluate;

$$
\begin{aligned}
I_{j+2}^{\mu} & =\frac{1}{\mathcal{N}} \int_{0}^{\infty} d \chi \chi \int_{-\pi}^{\pi} d \theta e^{i|\boldsymbol{b}| \chi \cos \theta} q^{\mu} \frac{1}{\chi}\left(\frac{\chi^{2}}{2}\right)^{j+2} \\
& =\frac{2 \pi i}{\mathcal{N}} \int_{0}^{\infty} d \chi \chi J_{1}(|\boldsymbol{b}| \chi) \hat{\boldsymbol{b}}^{\mu}\left(\frac{\chi^{2}}{2}\right)^{j+2} \\
& =\frac{\pi i}{8 m_{1} m_{2} \sqrt{\omega^{2}-1}} \frac{b^{\mu}}{|\boldsymbol{b}|}\left(-\frac{2}{b^{2}}\right)^{j+3} \frac{\Gamma[7 / 2+j]}{\Gamma[-3 / 2-j]}
\end{aligned}
$$


Next, we have the rank-2 integral, which must take the form

$$
I_{j+2}^{\mu \nu}=\alpha_{2} b^{\mu} b^{\mu}+\beta_{2} \Pi^{\mu \nu}
$$

where

$$
\Pi_{\nu}^{\mu}=\delta_{\nu}^{\mu}+\frac{1}{\omega^{2}-1}\left(v_{1}^{\mu}\left(v_{1 \nu}-\omega v_{2 \nu}\right)+v_{2}^{\mu}\left(v_{2 \nu}-\omega v_{1 \nu}\right)\right)
$$

is the projector onto the 2-dimensional plane perpendicular to $v_{1}$ and $v_{2}[6]$. By taking the trace of eq. (B.9), we have that

$$
\begin{aligned}
\alpha_{2} b^{2}+2 \beta_{2} & =-\frac{1}{\mathcal{N}} \int_{0}^{\infty} d \chi \chi \int_{-\pi}^{\pi} d \theta e^{i|\boldsymbol{b}| \boldsymbol{q} \cos \theta} \chi\left(\frac{\chi^{2}}{2}\right)^{j+2} \\
& =-\frac{4 \pi}{\mathcal{N}} \int_{0}^{\infty} d \chi J_{0}(|\boldsymbol{b}| \chi)\left(\frac{\chi^{2}}{2}\right)^{j+3} \\
& =-\frac{4 \pi}{\mathcal{N}} \frac{1}{|\boldsymbol{b}|}\left(-\frac{2}{b^{2}}\right)^{j+3} \frac{\Gamma[7 / 2+j]}{\Gamma[-5 / 2-j]}
\end{aligned}
$$

If we contract with $b_{\mu} b_{\nu}$ instead, we find that

$$
\begin{aligned}
\alpha_{2} b^{4}+\beta_{2} b^{2} & =-\frac{b^{2}}{\mathcal{N}} \int_{0}^{\infty} d \chi \chi^{2} \int_{-\pi}^{\pi} d \theta e^{i|\boldsymbol{b}| \chi \cos \theta}(\cos \theta)^{2}\left(\frac{\chi^{2}}{2}\right)^{j+2} \\
& =-\frac{4 \pi b^{2}}{\mathcal{N}} \int_{0}^{\infty} d \chi\left(J_{1}(|\boldsymbol{b}| \chi) \frac{1}{|\boldsymbol{b}| \chi}-J_{2}(|\boldsymbol{b}| \chi)\right)\left(\frac{\chi^{2}}{2}\right)^{j+3} \\
& =\frac{4 \pi b^{2}}{\mathcal{N}} \frac{1}{|\boldsymbol{b}|}\left(-\frac{2}{b^{2}}\right)^{j+3} \frac{(j+3) \Gamma[7 / 2+j]}{\Gamma[-3 / 2-j]}
\end{aligned}
$$

Putting this together with eq. (B.11), we end up with

$$
I_{j+2}^{\mu \nu}=\left((7+2 j) \frac{b^{\mu} b^{\nu}}{b^{2}}-\Pi^{\mu \nu}\right) \frac{\pi}{8 m_{1} m_{2} \sqrt{\omega^{2}-1}} \frac{1}{|\boldsymbol{b}|}\left(-\frac{2}{b^{2}}\right)^{j+3} \frac{\Gamma[7 / 2+j]}{\Gamma[-3 / 2-j]} .
$$

Lastly, the rank-3 integral is

$$
I_{j+2}^{\mu \nu \rho}=\alpha_{3} b^{\mu} b^{\nu} b^{\rho}+\beta_{3} b^{(\mu} \Pi^{\nu \rho)}
$$

(with normalization $1 / 3$ ! for the second term). Contracting with $b_{\mu} \eta_{\nu \rho}$, we have that

$$
\begin{aligned}
\alpha_{3} b^{4}+\beta_{3} \frac{4}{3} b^{2} & =\frac{1}{\mathcal{N}} \int_{0}^{\infty} d \chi \chi \int_{-\pi}^{\pi} d \theta e^{i|\boldsymbol{b}| \chi \cos \theta}|\boldsymbol{b}| \chi^{2} \cos \theta\left(\frac{\chi^{2}}{2}\right)^{j+2} \\
& =\frac{4 \pi i|\boldsymbol{b}|}{\mathcal{N}} \int_{0}^{\infty} d \chi J_{1}(|\boldsymbol{b}| \chi) \chi\left(\frac{\chi^{2}}{2}\right)^{j+3} \\
& =\frac{8 \pi i}{\mathcal{N}} \frac{1}{|\boldsymbol{b}|}\left(\frac{2}{\boldsymbol{b}^{2}}\right)^{j+3} \frac{\Gamma[9 / 2+j]}{\Gamma[-5 / 2-j]}
\end{aligned}
$$


When we contract with $b_{\mu} b_{\nu} b_{\rho}$, we find that

$$
\begin{aligned}
\alpha_{3} b^{6}+\beta b^{4} & =-\frac{1}{\mathcal{N}} \int_{0}^{\infty} d \chi \chi \int_{-\pi}^{\pi} d \theta e^{i|\boldsymbol{b}| \chi \cos \theta}(|\boldsymbol{b}| \chi \cos \theta)^{3} \frac{1}{\chi}\left(\frac{\chi^{2}}{2}\right)^{j+2} \\
& =-\frac{2 \pi i}{\mathcal{N}}|\boldsymbol{b}|^{3} \int_{0}^{\infty} d \chi\left(J_{2}(|\boldsymbol{b}| \chi) \frac{3}{|\boldsymbol{b}| \chi}-J_{3}(\mid \boldsymbol{b} \chi)\right) \chi^{3}\left(\frac{\chi^{2}}{2}\right)^{j+2} \\
& =-\frac{8 \pi i b^{2}}{\mathcal{N}} \frac{1}{|\boldsymbol{b}|}\left(-\frac{2}{b^{2}}\right)^{j+3} \frac{(j+3) \Gamma[9 / 2+j]}{\Gamma[-3 / 2-j]} .
\end{aligned}
$$

The integral becomes

$$
I_{j+2}^{\mu \nu \rho}=\left((9 / 2+j) \frac{b^{\mu} b^{\nu} b^{\rho}}{b^{2}}-\frac{3}{2} b^{(\mu} \Pi^{\nu \rho)}\right) \frac{i \pi}{4 m_{1} m_{2} \sqrt{\omega^{2}-1}} \frac{1}{|\boldsymbol{b}|}\left(-\frac{2}{b^{2}}\right)^{j+4} \frac{\Gamma[9 / 2+j]}{\Gamma[-3 / 2-j]} .
$$

We also need these integrals restricted to spatial indices. We can write the spatial portions of the integrals in as

$$
I_{j+2}^{i_{1} \ldots i_{n}}=(-i)^{n} \frac{\partial^{n}}{\partial b^{i_{1}} \ldots \partial b^{i_{n}}} I_{j+2}
$$

Computing derivatives of the rank 0 integral gives

$$
\begin{aligned}
I_{j+2}^{m} & =\frac{\pi i}{8 m_{1} m_{2} \sqrt{\omega^{2}-1}} \frac{\boldsymbol{b}^{m}}{|\boldsymbol{b}|}\left(\frac{2}{\boldsymbol{b}^{2}}\right)^{j+3} \frac{\Gamma[7 / 2+j]}{\Gamma[-3 / 2-j]}, \\
I_{j+2}^{m n} & =\left(\Pi^{m n}-(7+2 j) \frac{\boldsymbol{b}^{m} \boldsymbol{b}^{n}}{\boldsymbol{b}^{2}}\right) \frac{\pi}{8 m_{1} m_{2} \sqrt{\omega^{2}-1}} \frac{1}{|\boldsymbol{b}|}\left(\frac{2}{\boldsymbol{b}^{2}}\right)^{j+3} \frac{\Gamma[7 / 2+j]}{\Gamma[-3 / 2-j]}, \\
I_{j+2}^{m n l} & =\left(\frac{3}{2} \boldsymbol{b}^{(m} \Pi^{n l)}-(9 / 2+j) \frac{\boldsymbol{b}^{m} \boldsymbol{b}^{n} \boldsymbol{b}^{l}}{\boldsymbol{b}^{2}}\right) \frac{i \pi}{4 m_{1} m_{2} \sqrt{\omega^{2}-1}} \frac{1}{|\boldsymbol{b}|}\left(\frac{2}{\boldsymbol{b}^{2}}\right)^{j+4} \frac{\Gamma[9 / 2+j]}{\Gamma[-3 / 2-j]} .
\end{aligned}
$$

Moreover, note that the projector to the plane perpendicular to the velocities must be modified when restricted to purely spatial components:

$$
\Pi^{m n}=\delta^{m n}-\frac{1}{\omega^{2}-1}\left[\boldsymbol{v}_{1}^{m}\left(\boldsymbol{v}_{1}^{n}-\omega \boldsymbol{v}_{2}^{n}\right)+\boldsymbol{v}_{2}^{m}\left(\boldsymbol{v}_{2}^{n}-\omega \boldsymbol{v}_{1}^{n}\right)\right] .
$$

\section{B.3 Eikonal operator}

The eikonal phase was written in section 6 as the action of the operator $\hat{\mathcal{K}}_{j}$ on the integral $I_{j+2}$. We list here for reference the action of each term in this operator on this integral:

$$
\begin{aligned}
\hat{\mathcal{O}}^{(0)} I_{j+2} & =I_{j+2}, \\
\hat{\mathcal{O}}^{(1, a)} I_{j+2} & =-i\left(\boldsymbol{S}_{a} \times \boldsymbol{p}\right)_{m} I_{j+2}^{m}, \\
\hat{\mathcal{O}}^{(2,1)} I_{j+2} & =\boldsymbol{S}_{1 m} \boldsymbol{S}_{2 n} I_{j+2}^{m n}, \\
\hat{\mathcal{O}}^{(2,2)} I_{j+2} & =\left(\boldsymbol{S}_{1} \cdot \boldsymbol{S}_{2}\right) \delta_{m n} I_{j+2}^{m n}=2\left(\boldsymbol{S}_{1} \cdot \boldsymbol{S}_{2}\right) I_{j+3}, \\
\hat{\mathcal{O}}^{(2,3)} I_{j+2} & =\left(\boldsymbol{p} \cdot \boldsymbol{S}_{1}\right)\left(\boldsymbol{p} \cdot \boldsymbol{S}_{2}\right) \delta_{m n} I_{j+2}^{m n}=2\left(\boldsymbol{p} \cdot \boldsymbol{S}_{1}\right)\left(\boldsymbol{p} \cdot \boldsymbol{S}_{2}\right) I_{j+3},
\end{aligned}
$$

where $m, n$ are spatial indices. 
Open Access. This article is distributed under the terms of the Creative Commons Attribution License (CC-BY 4.0), which permits any use, distribution and reproduction in any medium, provided the original author(s) and source are credited.

\section{References}

[1] Ligo Scientific and Virgo collaborations, Observation of Gravitational Waves from a Binary Black Hole Merger, Phys. Rev. Lett. 116 (2016) 061102 [arXiv:1602.03837] [INSPIRE].

[2] A. Guevara, Holomorphic Classical Limit for Spin Effects in Gravitational and Electromagnetic Scattering, JHEP 04 (2019) 033 [arXiv: 1706.02314] [INSPIRE].

[3] C. Cheung, I.Z. Rothstein and M.P. Solon, From Scattering Amplitudes to Classical Potentials in the Post-Minkowskian Expansion, Phys. Rev. Lett. 121 (2018) 251101 [arXiv: 1808.02489] [INSPIRE].

[4] D.A. Kosower, B. Maybee and D. O'Connell, Amplitudes, Observables, and Classical Scattering, JHEP 02 (2019) 137 [arXiv: 1811.10950] [INSPIRE].

[5] A. Cristofoli, N.E.J. Bjerrum-Bohr, P.H. Damgaard and P. Vanhove, Post-Minkowskian Hamiltonians in general relativity, Phys. Rev. D 100 (2019) 084040 [arXiv:1906.01579] [INSPIRE].

[6] B. Maybee, D. O'Connell and J. Vines, Observables and amplitudes for spinning particles and black holes, JHEP 12 (2019) 156 [arXiv:1906.09260] [INSPIRE].

[7] N.E.J. Bjerrum-Bohr, A. Cristofoli and P.H. Damgaard, Post-Minkowskian Scattering Angle in Einstein Gravity, JHEP 08 (2020) 038 [arXiv:1910.09366] [INSPIRE].

[8] Z. Bern, A. Luna, R. Roiban, C.-H. Shen and M. Zeng, Spinning Black Hole Binary Dynamics, Scattering Amplitudes and Effective Field Theory, arXiv:2005.03071 [INSPIRE].

[9] G. Mogull, J. Plefka and J. Steinhoff, Classical black hole scattering from a worldline quantum field theory, JHEP 02 (2021) 048 [arXiv: 2010.02865] [INSPIRE].

[10] G. Kälin and R.A. Porto, From Boundary Data to Bound States, JHEP 01 (2020) 072 [arXiv: 1910.03008] [INSPIRE].

[11] G. Kälin and R.A. Porto, From boundary data to bound states. Part II. Scattering angle to dynamical invariants (with twist), JHEP 02 (2020) 120 [arXiv: 1911.09130] [INSPIRE].

[12] A. Guevara, A. Ochirov and J. Vines, Scattering of Spinning Black Holes from Exponentiated Soft Factors, JHEP 09 (2019) 056 [arXiv: 1812.06895] [INSPIRE].

[13] M.-Z. Chung, Y.-T. Huang, J.-W. Kim and S. Lee, The simplest massive S-matrix: from minimal coupling to Black Holes, JHEP 04 (2019) 156 [arXiv:1812.08752] [INSPIRE].

[14] A. Guevara, A. Ochirov and J. Vines, Black-hole scattering with general spin directions from minimal-coupling amplitudes, Phys. Rev. D 100 (2019) 104024 [arXiv:1906.10071] [INSPIRE].

[15] N. Arkani-Hamed, Y.-t. Huang and D. O'Connell, Kerr black holes as elementary particles, JHEP 01 (2020) 046 [arXiv: 1906.10100] [INSPIRE].

[16] M.-Z. Chung, Y.-T. Huang and J.-W. Kim, Classical potential for general spinning bodies, JHEP 09 (2020) 074 [arXiv: 1908. 08463] [INSPIRE]. 
[17] P.H. Damgaard, K. Haddad and A. Helset, Heavy Black Hole Effective Theory, JHEP 11 (2019) 070 [arXiv : 1908.10308] [inSPIRE].

[18] R. Aoude, K. Haddad and A. Helset, On-shell heavy particle effective theories, JHEP 05 (2020) 051 [arXiv: 2001.09164] [INSPIRE].

[19] M.-Z. Chung, Y.-t. Huang, J.-W. Kim and S. Lee, Complete Hamiltonian for spinning binary systems at first post-Minkowskian order, JHEP 05 (2020) 105 [arXiv: 2003. 06600] [INSPIRE].

[20] Z. Bern, C. Cheung, R. Roiban, C.-H. Shen, M.P. Solon and M. Zeng, Scattering Amplitudes and the Conservative Hamiltonian for Binary Systems at Third Post-Minkowskian Order, Phys. Rev. Lett. 122 (2019) 201603 [arXiv:1901.04424] [INSPIRE].

[21] Z. Bern, C. Cheung, R. Roiban, C.-H. Shen, M.P. Solon and M. Zeng, Black Hole Binary Dynamics from the Double Copy and Effective Theory, JHEP 10 (2019) 206 [arXiv: 1908.01493] [INSPIRE].

[22] C. Cheung and M.P. Solon, Classical gravitational scattering at $\mathcal{O}\left(G^{3}\right)$ from Feynman diagrams, JHEP 06 (2020) 144 [arXiv: 2003.08351] [INSPIRE].

[23] G. Kälin, Z. Liu and R.A. Porto, Conservative Dynamics of Binary Systems to Third Post-Minkowskian Order from the Effective Field Theory Approach, Phys. Rev. Lett. 125 (2020) 261103 [arXiv: 2007. 04977] [InSPIRE].

[24] T. Binnington and E. Poisson, Relativistic theory of tidal Love numbers, Phys. Rev. D 80 (2009) 084018 [arXiv: 0906.1366] [INSPIRE].

[25] T. Damour and A. Nagar, Relativistic tidal properties of neutron stars, Phys. Rev. D 80 (2009) 084035 [arXiv:0906.0096] [inSPIRE].

[26] B. Kol and M. Smolkin, Black hole stereotyping: Induced gravito-static polarization, JHEP 02 (2012) 010 [arXiv: 1110.3764] [INSPIRE].

[27] N. Gürlebeck, No-hair theorem for Black Holes in Astrophysical Environments, Phys. Rev. Lett. 114 (2015) 151102 [arXiv: 1503.03240] [INSPIRE].

[28] P. Pani, L. Gualtieri, A. Maselli and V. Ferrari, Tidal deformations of a spinning compact object, Phys. Rev. D 92 (2015) 024010 [arXiv: 1503.07365] [InSPIRE].

[29] P. Landry and E. Poisson, Tidal deformation of a slowly rotating material body. External metric, Phys. Rev. D 91 (2015) 104018 [arXiv:1503.07366] [InSPIRE].

[30] A. Le Tiec and M. Casals, Spinning Black Holes Fall in Love, arXiv:2007.00214 [INSPIRE].

[31] A. Le Tiec, M. Casals and E. Franzin, Tidal Love Numbers of Kerr Black Holes, arXiv:2010.15795 [INSPIRE].

[32] LIGO ScIentific and ViRgo collaborations, GW170817: Observation of Gravitational Waves from a Binary Neutron Star Inspiral, Phys. Rev. Lett. 119 (2017) 161101 [arXiv: 1710.05832] [INSPIRE].

[33] LIGO ScientifiC and ViRgo collaborations, GW170817: Measurements of neutron star radii and equation of state, Phys. Rev. Lett. 121 (2018) 161101 [arXiv:1805.11581] [INSPIRE].

[34] C. Cheung and M.P. Solon, Tidal Effects in the Post-Minkowskian Expansion, Phys. Rev. Lett. 125 (2020) 191601 [arXiv: 2006. 06665] [INSPIRE].

[35] K. Haddad and A. Helset, Tidal effects in quantum field theory, JHEP 12 (2020) 024 [arXiv:2008.04920] [INSPIRE]. 
[36] C. Cheung, N. Shah and M.P. Solon, Mining the Geodesic Equation for Scattering Data, Phys. Rev. D 103 (2021) 024030 [arXiv: 2010.08568] [InSPIRE].

[37] Z. Bern, J. Parra-Martinez, R. Roiban, E. Sawyer and C.-H. Shen, Leading Nonlinear Tidal Effects and Scattering Amplitudes, arXiv:2010.08559 [INSPIRE].

[38] D. Bini, T. Damour and A. Geralico, Scattering of tidally interacting bodies in post-Minkowskian gravity, Phys. Rev. D 101 (2020) 044039 [arXiv:2001.00352] [INSPIRE].

[39] G. Kälin and R.A. Porto, Post-Minkowskian Effective Field Theory for Conservative Binary Dynamics, JHEP 11 (2020) 106 [arXiv:2006.01184] [inSPIRE].

[40] G. Kälin, Z. Liu and R.A. Porto, Conservative Tidal Effects in Compact Binary Systems to Next-to-Leading Post-Minkowskian Order, Phys. Rev. D 102 (2020) 124025 [arXiv: 2008.06047] [INSPIRE].

[41] M. Levi, A.J. Mcleod and M. Von Hippel, NNNLO gravitational quadratic-in-spin interactions at the quartic order in $G$, arXiv:2003.07890 [INSPIRE].

[42] M. Levi and F. Teng, NLO gravitational quartic-in-spin interaction, JHEP 01 (2021) 066 [arXiv:2008.12280] [INSPIRE].

[43] M. Levi and J. Steinhoff, Spinning gravitating objects in the effective field theory in the post-Newtonian scheme, JHEP 09 (2015) 219 [arXiv:1501.04956] [INSPIRE].

[44] P.K. Gupta, J. Steinhoff and T. Hinderer, Relativistic effective action of dynamical gravitomagnetic tides for slowly rotating neutron stars, Phys. Rev. Res. 3 (2021) 013147 [arXiv: 2011.03508] [INSPIRE].

[45] L. Lehman and A. Martin, Hilbert Series for Constructing Lagrangians: expanding the phenomenologist's toolbox, Phys. Rev. D 91 (2015) 105014 [arXiv:1503.07537] [INSPIRE].

[46] L. Lehman and A. Martin, Low-derivative operators of the Standard Model effective field theory via Hilbert series methods, JHEP 02 (2016) 081 [arXiv: 1510.00372] [INSPIRE].

[47] B. Henning, X. Lu, T. Melia and H. Murayama, 2, 84, 30, 993, 560, 15456, 11962, 261485, ...: Higher dimension operators in the SM EFT, JHEP 08 (2017) 016 [Erratum ibid. 09 (2019) 019] [arXiv: 1512.03433] [INSPIRE].

[48] B. Henning, X. Lu, T. Melia and H. Murayama, Hilbert series and operator bases with derivatives in effective field theories, Commun. Math. Phys. 347 (2016) 363 [arXiv: 1507.07240] [INSPIRE].

[49] B. Henning, X. Lu, T. Melia and H. Murayama, Operator bases, S-matrices, and their partition functions, JHEP 10 (2017) 199 [arXiv: 1706.08520] [INSPIRE].

[50] M. Ruhdorfer, J. Serra and A. Weiler, Effective Field Theory of Gravity to All Orders, JHEP 05 (2020) 083 [arXiv: 1908.08050] [INSPIRE].

[51] A. Kobach and S. Pal, Hilbert Series and Operator Basis for NRQED and NRQCD/HQET, Phys. Lett. B 772 (2017) 225 [arXiv: 1704.00008] [InSPIRE].

[52] A. Kobach and S. Pal, Conformal Structure of the Heavy Particle EFT Operator Basis, Phys. Lett. B $\mathbf{7 8 3}$ (2018) 311 [arXiv:1804.01534] [INSPIRE].

[53] L. Graf, B. Henning, X. Lu, T. Melia and H. Murayama, 2, 12, 117, 1959, 45171, 1170086, ...: a Hilbert series for the QCD chiral Lagrangian, JHEP 01 (2021) 142 [arXiv: 2009.01239] [INSPIRE]. 
[54] N. Arkani-Hamed, T.-C. Huang and Y.-t. Huang, Scattering Amplitudes For All Masses and Spins, arXiv:1709.04891 [INSPIRE].

[55] G. Durieux and C.S. Machado, Enumerating higher-dimensional operators with on-shell amplitudes, Phys. Rev. D 101 (2020) 095021 [arXiv: 1912.08827] [INSPIRE].

[56] G. Durieux, T. Kitahara, C.S. Machado, Y. Shadmi and Y. Weiss, Constructing massive on-shell contact terms, JHEP 12 (2020) 175 [arXiv: 2008.09652] [INSPIRE].

[57] H.-L. Li, Z. Ren, J. Shu, M.-L. Xiao, J.-H. Yu and Y.-H. Zheng, Complete Set of Dimension-8 Operators in the Standard Model Effective Field Theory, arXiv: 2005.00008 [INSPIRE].

[58] A. Falkowski, Bases of massless EFTs via momentum twistors, arXiv:1912.07865 [INSPIRE].

[59] K. Haddad and A. Helset, The double copy for heavy particles, Phys. Rev. Lett. 125 (2020) 181603 [arXiv: 2005.13897] [INSPIRE].

[60] W. Tulczyjew, Motion of multipole particles in general relativity theory binaries, Acta Phys. Polon. 18 (1959) 393.

[61] M. Levi, S. Mougiakakos and M. Vieira, Gravitational cubic-in-spin interaction at the next-to-leading post-Newtonian order, JHEP 01 (2021) 036 [arXiv:1912.06276] [INSPIRE].

[62] B.R. Holstein and A. Ross, Spin Effects in Long Range Electromagnetic Scattering, arXiv:0802.0715 [INSPIRE].

[63] B.R. Holstein and A. Ross, Spin Effects in Long Range Gravitational Scattering, arXiv:0802.0716 [INSPIRE].

[64] C. Lorcé, New explicit expressions for Dirac bilinears, Phys. Rev. D 97 (2018) 016005 [arXiv: 1705.08370] [INSPIRE].

[65] J. Vines, Scattering of two spinning black holes in post-Minkowskian gravity, to all orders in spin, and effective-one-body mappings, Class. Quant. Grav. 35 (2018) 084002 [arXiv: 1709.06016] [INSPIRE].

[66] D. Amati, M. Ciafaloni and G. Veneziano, Classical and Quantum Gravity Effects from Planckian Energy Superstring Collisions, Int. J. Mod. Phys. A 3 (1988) 1615 [InSPIRE].

[67] R. Aoude, M.-Z. Chung, Y.-t. Huang, C.S. Machado and M.-K. Tam, Silence of Binary Kerr Black Holes, Phys. Rev. Lett. 125 (2020) 181602 [arXiv:2007.09486] [INSPIRE].

[68] G. Passarino and M.J.G. Veltman, One Loop Corrections for $e^{+} e^{-}$Annihilation Into $\mu^{+} \mu^{-}$ in the Weinberg Model, Nucl. Phys. B 160 (1979) 151 [INSPIRE].

[69] V.A. Smirnov, Analytic tools for Feynman integrals, vol. 250, Springer (2012) [DOI] [INSPIRE]. 